\title{
CARACTERIZACIÓN SISMOTECTÓNICA DE LA REGIÓN DEL VALLE DEL CAUCA Y ZONAS ALEDAÑAS A PARTIR DE MECANISMOS FOCALES DE TERREMOTOS
}

Elkin De J. Salcedo-Hurtado ${ }^{1,2}$; John Leandro Pérez ${ }^{1,3}$

DOI: http://dx.doi.org/10.18273/revbol.v38n3-2016006 (ㄷ) (1) Ð

Forma de citar: Salcedo, E., y Pérez J.L. 2016. Caracterización sismotectónica de la región del Valle del Cauca y zonas aledañas a partir de mecanismos focales de terremotos. Boletín de Geología, 38(3): 89-107.

\section{RESUMEN}

El presente trabajo analiza el mecanismo focal de 49 terremotos con magnitud Mw $\geq 4,8$ en la región del Valle del Cauca y zonas aledañas, durante el periodo 1978-2010. Las soluciones fueron tomadas de los catálogos del Centro Internacional de Sismología (ISC, sigla en inglés) y del Centride Tensor de Momento Sísmico (GCMT, sigla en inglés). La profundidad de los eventos se distribuye entre superficiales e intermedios, hasta $215 \mathrm{~km}$ aproximadamente, asociados a la subducción de la placa Nazca, y unos pocos eventos superficiales que corresponden a la actividad de fuentes corticales en la placa continental Sudamericana. Concordante con el modelo de ambientes de regímenes de esfuerzos asociados con la deformación "estática" de la placa en subducción, se identifican cuatros ambientes sismotectónicos, que se distinguen por sus propias características sismológicas, tectónicas y régimen de esfuerzos. Se establece la tasa de deformación sismotectónica para cada uno de estos ambientes. El modelamiento del campo de esfuerzos de Coulomb muestra que el esfuerzo de cizalla crea una barrera que impide que el esfuerzo compresivo se proyecte al interior del continente en dirección SW-NE, interrumpiéndose en la zona en la fosa Colombo-Ecuatoriana. Mientras que el esfuerzo de tensión, aunque también es interrumpido, logra proyectarse al continente en dirección NW-SE, perpendicular al esfuerzo compresivo.

Palabras claves: Sismotectónica, mecanismos focales, terremotos, campo de esfuerzos.

\section{SEISMOTECTONIC CHARACTERIZATION OF THE CAUCA VALLEY REGION AND SURROUNDING AREAS FROM THE EARTHQUAKE FOCAL MECHANISMS}

\begin{abstract}
This paper analyzes the focal mechanism of 49 earthquakes with magnitude $\mathrm{Mw} \geq 4.8$ in the region of Valle del Cauca and surrounding areas during the period 1978-2010. The solutions were taken from the catalogs of the International Seismological Centre (ISC) and The Global Centroid-Moment-Tensor (GCMT) Project. The depth of the events is distributed between surface and intermediate about $215 \mathrm{~km}$, associated with the subduction of the Nazca plate, and a few superficial events that correspond to the activity of cortical sources in the South American continental plate. In accord with model environments strong regimes associated with the "static" deformation of the subducting plate, four seismotectonic environments, are distinguished by its own seismic, tectonic and strong regime characteristics. The seismotectonic deformation rate is presented for each of these environments. The modeling of the Coulomb effort field shows that the shear creates a barrier that prevents the compressive effort is projected into the continent in SW-NE direction, interrupted in the Colombo-Ecuadorian trench. While the tensile effort, although is also interrupted, it manages to project the continent in NW-SE direction, perpendicular to compressive effort.
\end{abstract}

Key words: Seismotectonic, focal mechanisms, earthquakes, stress field.

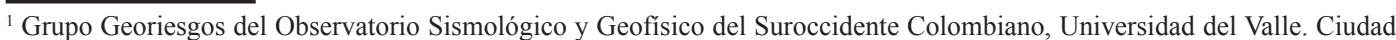
universitaria. Cali-Colombia. A. A. 25360. elkin.salcedo@correounivalle.edu.co

${ }^{2}$ Universidad del Valle, Departamento de Geografía, Ciudad universitaria. Calle 100 No. 13-00. Cali-Colombia.

${ }^{3}$ Posgrado en Ciencias de la Tierra, Centro de Investigación Científica y Educación Superior de Ensenada (CICESE), Baja California.
} 


\section{INTRODUCCIÓN}

La solución del mecanismo focal consiste en determinar los procesos físicos que han tenido lugar en la región del foco de un terremoto, permitiendo con ello obtener el correspondiente estado de esfuerzos que lo produce (Aki and Richards, 1980; Buforn, 1994; Udías y Mezcua, 1997; Udías et al., 1985; Baumbach and Grosser, 2009; Bormann and Wendt, 2013). La representación gráfica del mecanismo focal del terremoto puede ser obtenido, por ejemplo, a partir de los arribos de la onda $\mathrm{P}$ ó S de un sismograma (Buforn y Pro, 2006; Pro et al., 2007). El mecanismo focal se describe en términos de los tres ejes ortogonales del esfuerzo: eje $\mathrm{P}$ (compresivo), eje $\mathrm{T}$ (de tensión) y eje $\mathrm{N}$ (nulo); y tres ángulos de orientación del plano de falla: rumbo (Strike), buzamiento (Dip) y dirección de deslizamiento (Dip-Slip). El plano de falla es de interés para la geología estructural, por esta razón, la aplicación de los resultados de los mecanismos focales son de utilidad tanto en tectónica regional como para el conocimiento de las estructuras locales (Buforn y Pro, 2006; Cronin, 2010).

Tradicionalmente, las soluciones de los mecanismos focales de los terremotos han sido una de las principales herramientas para el análisis sismotectónico de regiones continentales y zonas oceánicas, como lo muestran trabajos realizados en diferentes regiones del mundo: zonas de rift en el océano y el manto (Sykes, 1967; Isaacks et al., 1969; Isaacks and Molnar, 1969), Centroamerica y el Caribe (Molnar and Sykes, 1969, Lòpez, 2012), en el Medio Oriente (Nowroozi, 1972), la región de Azores-Alborán (Udías et al., 1976; Mezcua et al., 1991); en el Mediterraneo (Jackson and McKenzie, 1988), regiones continentales (Ekstrom and England, 1989), la región de Tayikistan (Sobolieva, 1990), el arco de islas Kuriles, Kamchatka y Aleutianas (Voronina et al., 1990), en Grecia Central (Papazachos and Kiratzi, 1992), el Mar Andaman (Guzmán-Speziale and Ni, 1993), el norte y el este de la falla Anatolia (Kiratzi, 1993), Japón (Kiratzi and Papazachos, 1996), las fosas tectónicas de América Central (Guzmán-Speziale, 2001), el arco volcánico de América Central relacionado con la subducción de la placa de Cocos (Guzmán-Speziale et al., 2005), Panamá (Camacho et al., 2010), en la zona de colisión del Arco de Panamá con la esquina del noroccidente de Suramérica (Vargas and Mann, 2013), Nuevo Madrid (Johnson et al., 2014), entre otros.

Entre los principales trabajos aplicados al territorio colombiano se pueden encontrar los realizados por Mendiguren (1973), Bune et al. (1975), Jordan (1975),
Herd et al. (1981), Pennington (1981), Kanamori and McNally (1982), Mendoza and Dewey (1984), Lomnitz and Hashimine (1985), Page (1986), Adamek et al. (1988), Rivera (1989), Salcedo (1992), Freymueller et al. (1993), Salcedo (1995), Salcedo et al. (1995), Tabares et al. (1999), Salcedo et al. (2001), Trenkamp et al. (2002), Pulido (2003), Corredor (2003), Monsalve y Mora (2005), Cardona et al. (2005), Cortés and Angelier (2005), Pedraza (2006), Vargas and Mann (2013), entre otros.

El presente trabajo muestra los resultados de la búsqueda de las soluciones del mecanismo focal para una población de terremotos ocurridos en el periodo comprendido entre 1978 a 2010, con magnitud $M w \geq$ 4,8. Los datos de las soluciones del mecanismo focal fueron obtenidos del catálogo del ISC (International Seismological Center) y del GCMT (Global Centroid Moment Tensor), a partir de los cuales se realiza el análisis del marco sismotectónico de la región del Valle del Cauca y zonas aledañas.

\section{MARCO TECTÓNICO Y SISMICIDAD}

La región del Valle del Cauca se ubica en el suroccidente colombiano, donde la placa Nazca (corteza oceánica), que se separa de la placa del Pacífico a través de una zona de divergencia o rift, converge y subduce por debajo de la placa de Sudamérica. La placa Nazca se desplaza en dirección W-E con movimiento relativo entre $50 \mathrm{~mm} / \mathrm{año}$ y $78 \mathrm{~mm} /$ año (Pennington, 1981; Kellogg et al., 1989; Freymueller et al., 1993; Gutscher et al., 1999; Trenkamp et al., 2002, Trenkamp et al., 2004), conservando sus propiedades mecánicas hasta ser consumida en el manto debajo del continente suramericano. El límite activo entre las placas de Nazca y Sudamérica está definido por la fosa ColomboEcuatoriana que recorre el fondo marino más o menos paralelo a la costa y define el límite superficial de la zona de subducción (FIGURA 1).

El marco tectónico de la esquina noroccidental de Suramérica a lo largo de la fosa Colombo-Ecuatoriana, es dominado por la subducción de la placa oceánica bajo la continental, donde varios autores describen la existencia de al menos tres segmentos tectónicos sísmicamente activos (Pennington, 1981; Monsalve, 1998; Corredor, 2003; Collot et al., 2004; Arcila y Dimaté, 2005; Pedraza, 2006). Cada uno de estos segmentos: Norte, Centro y Sur, se caracteriza por tener su propio régimen sísmico y procesos de liberación y acumulación de esfuerzos de manera diferente (Corredor, 2003; Arcila y Dimaté, 2005; Pedraza, 2006). 


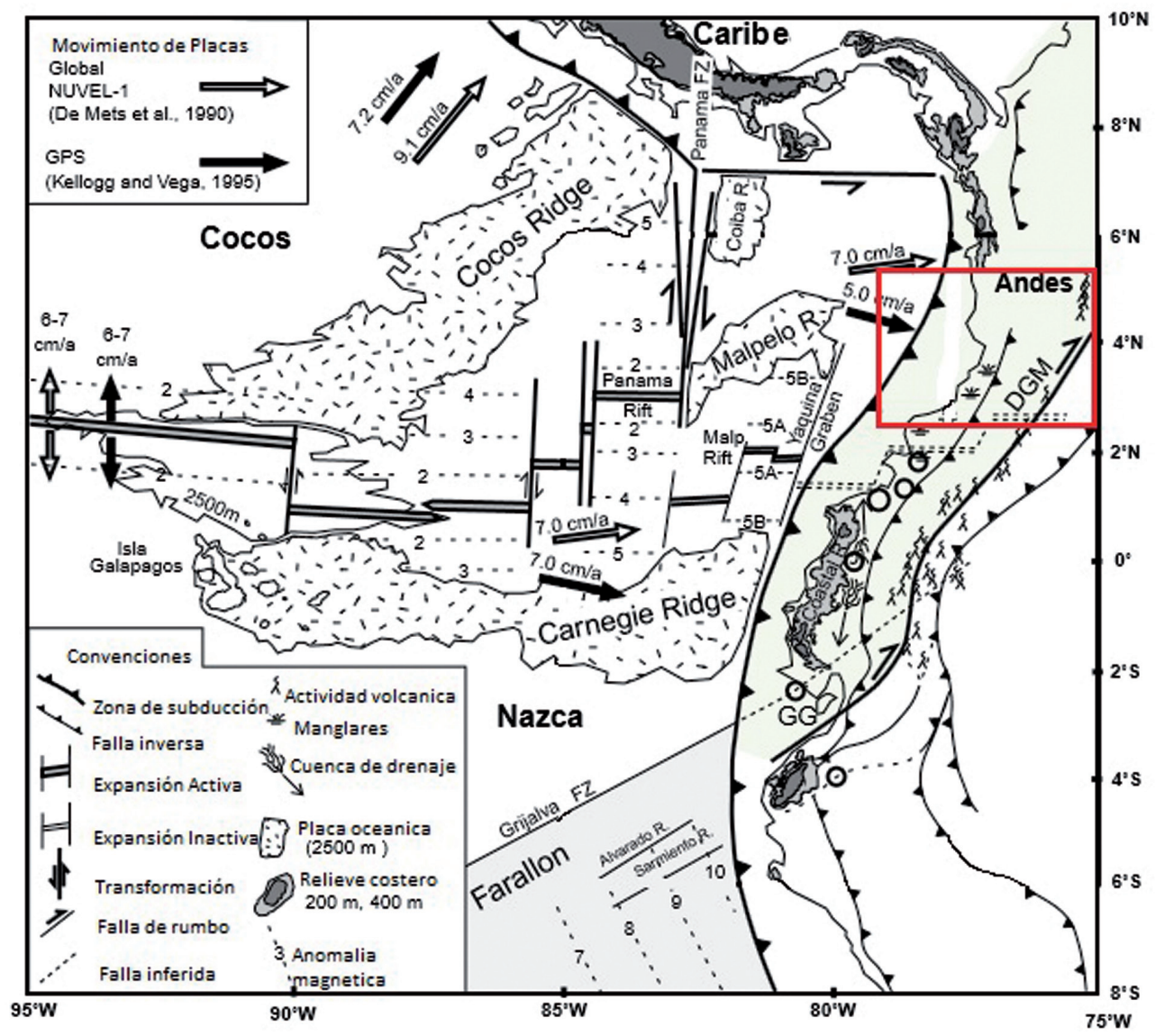

FIGURA 1. Características tectónicas de la zona de convergencia de Nazca y Sudamérica y movimiento relativo de las placas de acuerdo con los datos GPS. El recuadro de color rojo indica la zona de estudio del presente trabajo (Modificado de Gutscher et al., 1999).

De acuerdo con Arcila y Dimaté (2005), estos segmentos se describen de tal forma que: a) el "Segmento Norte", representa la subducción del bloque Coiba bajo el extremo noroeste de Colombia, aquí la fosa tiene una longitud de $170 \mathrm{~km}$. Este segmento está orientado con un azimut de $130^{\circ}$ cuyo plano de Benioff se inclina $25^{\circ}$ a $40^{\circ}$, la magnitud máxima esperada para este segmento es de 7,8 Mw; b) el "Segmento Centro", con una longitud en la fosa de $160 \mathrm{~km}$, se orienta con un azimut de $20^{\circ}$ y su plano de Benioff definido bajo el Viejo Caldas se inclina $40^{\circ}$ a $70^{\circ}$, la magnitud máxima esperada aquí también es de 7,8 Mw; y c) el "Segmento Sur" cuyo tramo de la fosa está orientado con un azimut $40^{\circ}$ y tiene una longitud de $550 \mathrm{~km}$. El plano de Benioff está inclinado $30^{\circ}$ a $50^{\circ}$ y la magnitud máxima calculada es $8,8 \mathrm{Mw}$.

Los segmentos Central y Sur son los que afecta en gran medida el departamento del Valle del Cauca y zonas aledañas. Particularmente, al Segmento Sur se le atribuyen importantes sismos frente a las costas del Pacífico entre Ecuador y Colombia como los de
$1906(8,9 M w), 1958(7,8 M w), 1942(5,8 M s), 1979$ $(8,1 \mathrm{Mw})$ (Kelleher, 1972; Ruff and Kanamori, 1980; Kanamori and McNally, 1982) y el más reciente de magnitud 7,8 Mw que generó grandes impactos en la población y las estructuras al norte de Ecuador y que se sintió fuertemente en el Suroccidente de Colombia. Desde el punto de vista sismotectónico, en el Valle del Cauca se revelan tres tipos de fuentes sismogénicas (INGEOMINAS, 2005): 1) la trinchera; 2) la zona de Benioff; y 3) las fallas intracontinentales, por ejemplo, el sistema de fallas Romeral y el sistema de fallas Cauca (FIGURA 2).

En estas fuentes sismogénicas se han presentado terremotos históricos fuertes con profundidades desde superficiales hasta intermedios, que han dejado considerables daños materiales y numerosas víctimas en el occidente y centro del país, entre los que se destacan los sismos: del 9 de Julio de 1766, cuyos daños más importantes se observaron en las ciudades de Buga y Cali, 31 de enero de 1906 con magnitud 8,9 $M w$ entre Colombia y Ecuador, que genero un tsunami 
cuyos efectos más graves fueron sobre la costa de los departamentos de Cauca y Nariño La ocurrencia de este evento se asocia a al segmento Tumaco, que según (Acosta et al., 2007), es una la gran zona de ruptura, diferenciada por el cambio geométrico a lo largo de la fosa entre las placas Nazca y Sudamericana, donde se han registrados otros de sismos de subducción de gran magnitud $M w$ 7,9 (1942) y $M w 7,8$ (1958). También se destacan los terremotos: de julio 30 de 1962 con magnitud 6,9 Ms en Caldas, 19 de diciembre de 1991 de 7,2 Mw. Estos dos últimos eventos generaron daños considerables en la infraestructura de la ciudad de Cali.

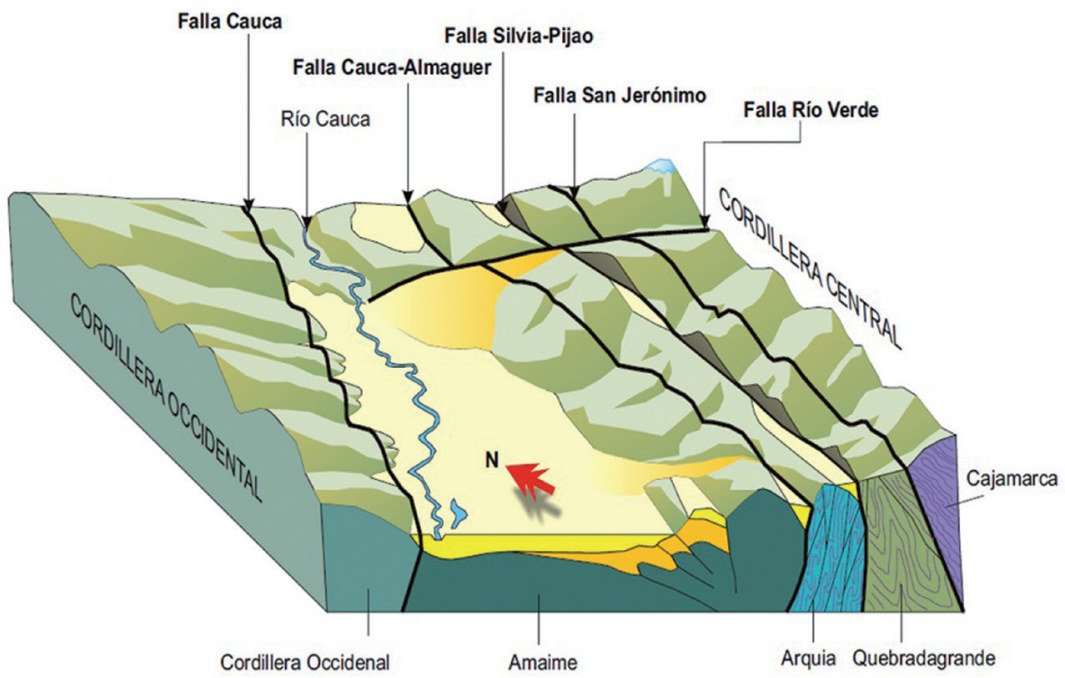

FIGURA 2. Esquema del sistema de fallas en la Cordillera Central. Al occidente del río Cauca, la Falla Cauca también conocida como Cauca-Patía y al oriente del río Cauca, las fallas Cauca-Almaguer, Silvia-Pijao y San Jerónimo. (Tomado de López, 2006).

Otros eventos recientes son los ocurridos: el 30 septiembre de 2012 con magnitud 7,1 Mw y epicentro cerca al municipio La Vega en el departamento del Cauca, 9 de febrero de 2013 con magnitud 6,9 Mw localizado cerca al municipio de Ospina (Cauca), y 13 de agosto de 2013 con magnitud 6,5 Mw, ubicado en Nuquí departamento del Choco y sentido en todo el occidente del país y en la región del Darién cerca a Panamá.

De los terremotos de fuentes corticales con mayores efectos en las regiones del occidente y centro del país, pueden destacarse: el del 7 de junio de 1925 con magnitud 6,8 Ms e intensidad VII-VIII (escala EMS98) en la ciudad de Cali, 23 de noviembre de 1979 con magnitud 7,2 Mw e intensidad VII (EMS-98) en Risaralda y efectos en Chocó y Valle del Cauca, 8 de febrero de 1995 con magnitud 6,4 Mw causando daños en edificaciones de los departamentos del Valle del Cauca y Risaralda, y 25 de enero de 1999 con magnitud 6,2 Mw dejando efectos devastadores en la ciudad de Armenia. En la FIGURA 3 se muestran los sismos históricos más importantes de la región.

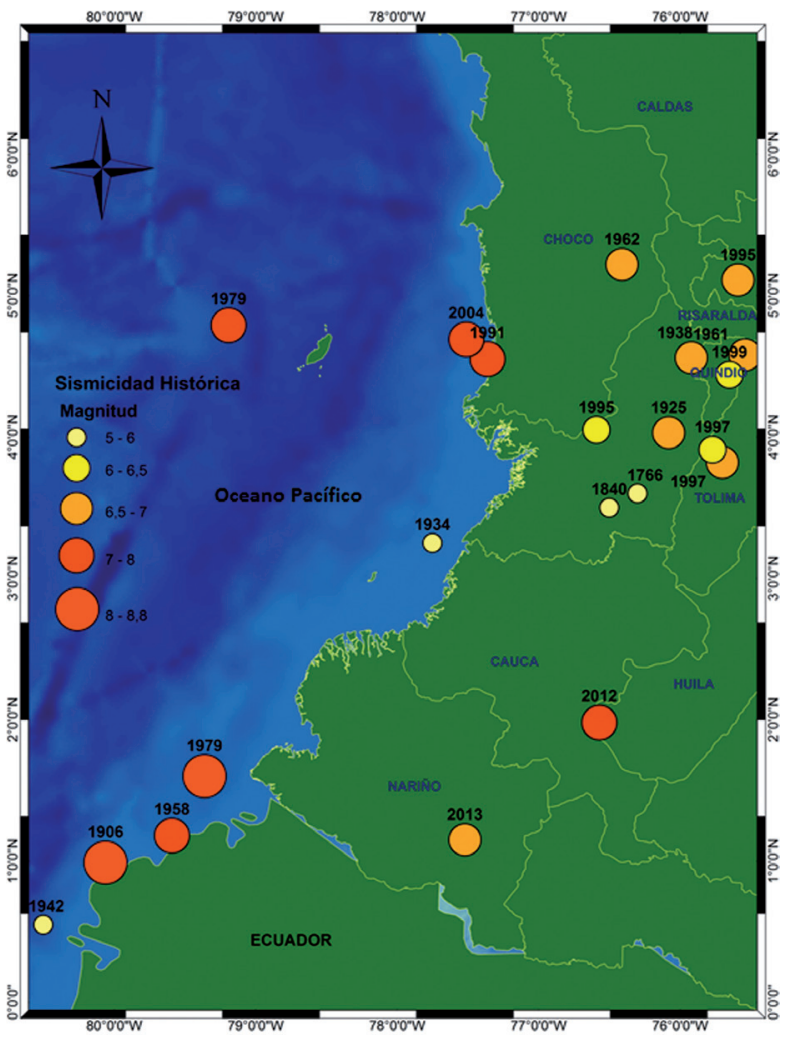

FIGURA 3. Localización de sismos históricos con efectos en la región del Suroccidente de Colombia. 


\section{DATOS Y MÉTODO}

Para este estudio se tomó la zona comprendida entre las coordenadas $2,5^{\circ}$ y $5,3^{\circ}$ de latitud norte y $75.6^{\circ}$ y $79.5^{\circ}$ de longitud oeste, cubriendo el departamento del Valle del Cauca y parte de los departamentos de Chocó, Quindío, Risaralda, Tolima y Cauca. Los datos de las soluciones del mecanismo focal se tomaron de los catálogos del ISC (International Seismological Center) y del GCMT (Global Centroid Moment Tensor) de la Universidad de Havard (Dziewonski et al., 1981), para sismos con magnitud $M w \geq 4,8$ ocurridos en el periodo comprendido entre 1978 a 2010, en total se reunieron 49 eventos con sus respectivas soluciones (TABLA 1).

TABLA 1. Sismos con magnitud $M w \geq 4,8$ con solución del mecanismo focal en la región del Valle del Cauca y zonas aledañas, ocurridos durante el periodo $1978-2010$.

\begin{tabular}{|c|c|c|c|c|c|c|c|c|c|}
\hline \multirow{2}{*}{ No. } & \multirow{2}{*}{$\begin{array}{c}\text { FECHA } \\
\mathbf{d} / \mathbf{m} / \mathbf{a} \\
\end{array}$} & \multirow{2}{*}{$\frac{\text { TIEMPO (UTC) }}{\text { hh:mm:ss }}$} & \multicolumn{3}{|c|}{ LOCALIZACIÓN } & \multicolumn{3}{|c|}{ Magnitud } & \multirow{2}{*}{$\frac{\text { Momento }(\mathrm{Mo})}{\text { dinas*cm }}$} \\
\hline & & & Latitud $\left({ }^{\circ} \mathbf{N}\right)$ & Longitud $\left({ }^{\circ} \mathrm{W}\right)$ & Prof. (Km) & Mw & mb & Ms & \\
\hline 1 & $16 / 02 / 1978$ & $3: 47: 12$ & 4,00 & 78,00 & 14,0 & 5,6 & 5,4 & 4,9 & $3,470 \mathrm{E}+24$ \\
\hline 2 & 29/05/1979 & $12: 59: 01$ & 5,22 & 75,80 & 122,0 & 5,0 & 4,9 & 0,0 & $4,520 \mathrm{E}+24$ \\
\hline 3 & 23/11/1979 & $23: 40: 30$ & 4,81 & 76,22 & 105,4 & 7,2 & 6,3 & 6,5 & $7,890 \mathrm{E}+24$ \\
\hline 4 & 13/12/1979 & 7:59:00 & 2,88 & 79,49 & 15,0 & 6,3 & 5,0 & 5,8 & $3,070 \mathrm{E}+24$ \\
\hline 5 & 07/01/1980 & $0: 33: 36$ & 2,94 & 78,77 & 30,8 & 5,3 & 5,0 & 0,0 & $9,510 \mathrm{E}+24$ \\
\hline 6 & 25/06/1980 & $12: 04: 57$ & 4,44 & 75,78 & 159,5 & 6,3 & 5,7 & 5,6 & $3,920 \mathrm{E}+24$ \\
\hline 7 & 01/07/1980 & $0: 33: 38$ & 2,93 & 78,75 & 19,0 & 5,3 & 0,0 & 0,0 & $9,510 \mathrm{E}+24$ \\
\hline 8 & 03/09/1980 & $22: 12: 39$ & 3,24 & 78,19 & 42,1 & 6,1 & 5,7 & 5,8 & $1,810 \mathrm{E}+24$ \\
\hline 9 & 08/01/1982 & $20: 38: 18$ & 2,57 & 78,87 & 25,1 & 5,3 & 0,0 & 0,0 & $1,070 \mathrm{E}+24$ \\
\hline 10 & $10 / 06 / 1985$ & $3: 23: 39$ & 3,24 & 78,99 & 26,0 & 5,5 & 5,5 & 4,9 & $2,520 \mathrm{E}+24$ \\
\hline 11 & $25 / 01 / 1987$ & $10: 31: 32$ & 3,30 & 79,31 & 15,4 & 5,7 & 5,6 & 5,2 & $5,140 \mathrm{E}+24$ \\
\hline 12 & 20/09/1988 & $17: 56: 25$ & 4,99 & 77,68 & 24,1 & 5,8 & 5,6 & 5,6 & $6,560 \mathrm{E}+24$ \\
\hline 13 & 29/11/1988 & $11: 23: 38$ & 4,72 & 77,07 & 75,0 & 5,7 & 5,6 & 0,0 & $3,860 \mathrm{E}+24$ \\
\hline 14 & $23 / 11 / 1990$ & $22: 35: 34$ & 4,73 & 75,60 & 138,0 & 6,1 & 0,0 & 0,0 & $1,110 \mathrm{E}+24$ \\
\hline 15 & 19/11/1991 & $23: 06: 58$ & 4,80 & 77,18 & 14,9 & 7,2 & 6,3 & 7,1 & $7,320 \mathrm{E}+24$ \\
\hline 16 & $10 / 12 / 1991$ & $22: 28: 01$ & 4,74 & 77,48 & 38,4 & 5,2 & 5,2 & 4,6 & $8,180 \mathrm{E}+24$ \\
\hline 17 & $15 / 08 / 1992$ & 19:02:09 & 5,11 & 75,61 & 124,0 & 5,9 & 5,6 & 0,0 & $7,300 \mathrm{E}+24$ \\
\hline 18 & $12 / 10 / 1993$ & $19: 51: 30$ & 4,15 & 76,98 & 102,9 & 5,2 & 5,5 & 0,0 & $7,540 \mathrm{E}+24$ \\
\hline 19 & 03/06/1994 & $11: 25: 07$ & 3,52 & 78,78 & 19,0 & 5,8 & 5,8 & 5,2 & $5,670 \mathrm{E}+24$ \\
\hline 20 & 06/06/1994 & $20: 47: 47$ & 2,93 & 75,94 & 32,6 & 6,8 & 6,3 & 6,7 & $1,840 \mathrm{E}+24$ \\
\hline 21 & $26 / 11 / 1994$ & 4:48:00 & 2,87 & 79,43 & 15,9 & 5,3 & 5,1 & 4,5 & $9,430 \mathrm{E}+24$ \\
\hline 22 & 08/02/1995 & $15: 05: 04$ & 4,10 & 76,62 & 77,1 & 6,4 & 6,2 & 5,6 & $4,090 \mathrm{E}+24$ \\
\hline 23 & $04 / 12 / 1995$ & $18: 40: 25$ & 2,72 & 78,32 & 49,4 & 5,1 & 5,2 & 4,6 & $4,360 \mathrm{E}+24$ \\
\hline 24 & $19 / 08 / 1995$ & $18: 22: 35$ & 5,22 & 75,69 & 128,7 & 6,5 & 6,2 & 5,9 & $7,720 \mathrm{E}+24$ \\
\hline 25 & $13 / 11 / 1995$ & $21: 43: 32$ & 2,83 & 79,44 & 15,0 & 5,3 & 5,3 & 4,5 & $1,280 \mathrm{E}+24$ \\
\hline 26 & $11 / 09 / 1996$ & $6: 28: 52$ & 4,59 & 76,90 & 118,2 & 5,3 & 5,0 & 0,0 & $1,110 \mathrm{E}+24$ \\
\hline 27 & $19 / 02 / 1997$ & $18: 25: 13$ & 4,56 & 76,49 & 105,0 & 5,8 & 5,5 & 0,0 & $5,800 \mathrm{E}+24$ \\
\hline 28 & 02/09/1997 & $12: 13: 23$ & 3,85 & 75,75 & 211,0 & 6,8 & 6,5 & 0,0 & $1,620 \mathrm{E}+24$ \\
\hline 29 & $11 / 12 / 1997$ & $7: 56: 29$ & 3,93 & 75,79 & 183,0 & 6,3 & 6,0 & 0,0 & $4,020 \mathrm{E}+24$ \\
\hline 30 & 25/01/1999 & $18: 19: 17$ & 4,58 & 75,75 & 27,7 & 6,1 & 5,9 & 5,7 & $2,010 \mathrm{E}+24$ \\
\hline 31 & 25/01/1999 & $22: 40: 23$ & 4,31 & 75,74 & 24,3 & 5,5 & 5,5 & 4,7 & $2,070 \mathrm{E}+24$ \\
\hline 32 & $22 / 09 / 2001$ & $3: 23: 38$ & 3,87 & 75,97 & 178,6 & 5,9 & 5,9 & 4,9 & $9,420 \mathrm{E}+24$ \\
\hline 33 & $02 / 07 / 2002$ & $16: 24: 29$ & 5,02 & 77,41 & 45,3 & 5,4 & 5,3 & 4,6 & $1,400 \mathrm{E}+24$ \\
\hline 34 & $08 / 08 / 2002$ & $13: 39: 58$ & 4,98 & 77,82 & 43,3 & 5,9 & 5,3 & 5,0 & $8,100 E+24$ \\
\hline 35 & $21 / 12 / 2002$ & $00: 46: 11$ & 3,76 & 78,90 & 15,0 & 5,2 & 5,3 & 4,5 & $9,230 \mathrm{E}+24$ \\
\hline 36 & $22 / 08 / 2003$ & $5: 29: 13$ & 3,07 & 77,73 & 31,4 & 5,0 & 5,0 & 4,2 & $3,560 \mathrm{E}+24$ \\
\hline 37 & 05/11/2003 & $00: 58: 51$ & 5,14 & 77,81 & 27,6 & 5,9 & 5,7 & 5,4 & $9,300 \mathrm{E}+24$ \\
\hline 38 & $15 / 11 / 2004$ & $9: 06: 56$ & 4,70 & 77,51 & 15,0 & 7,2 & 6,5 & 7,1 & $7,570 \mathrm{E}+24$ \\
\hline 39 & $08 / 03 / 2005$ & $15: 59: 43$ & 4,50 & 75,92 & 64,5 & 5,0 & 4,9 & 5,2 & $3,561 \mathrm{E}+24$ \\
\hline 40 & $21 / 04 / 2005$ & $3: 39: 24$ & 5,18 & 76,32 & 99,7 & 5,2 & 4,9 & 3,9 & $8,910 \mathrm{E}+24$ \\
\hline 41 & $03 / 08 / 2005$ & $15: 59: 43$ & 2,62 & 79,40 & 28,6 & 4,9 & 4,9 & 4,8 & $2,590 \mathrm{E}+24$ \\
\hline 42 & $17 / 03 / 2007$ & $22: 43: 09$ & 4,61 & 78,53 & 13,1 & 6,0 & 5,9 & 5,3 & $1,278 \mathrm{E}+24$ \\
\hline 43 & $18 / 03 / 2007$ & $2: 11: 05$ & 4,69 & 78,53 & 12,0 & 6,2 & 6,3 & 5,7 & $2,723 \mathrm{E}+24$ \\
\hline 44 & $10 / 09 / 2007$ & 1:49:14 & 3,08 & 78,12 & 18,9 & 6,7 & 6,0 & 6,7 & $1,662 \mathrm{E}+24$ \\
\hline 45 & $23 / 02 / 2008$ & $3: 23: 06$ & 4,06 & 78,75 & 12,0 & 5,1 & 4,8 & 4,4 & $5,334 \mathrm{E}+24$ \\
\hline 46 & $13 / 09 / 2008$ & 9:32:02 & 4,79 & 75,52 & 132,9 & 5,7 & 5,9 & 5,7 & $4,420 \mathrm{E}+24$ \\
\hline 47 & 08/09/2009 & $8: 26: 02$ & 4,77 & 76,71 & 82,7 & 4,8 & 5,0 & 0,0 & $2,080 \mathrm{E}+24$ \\
\hline 48 & $18 / 12 / 2009$ & $14: 30: 06$ & 3,08 & 76,30 & 169,2 & 5,1 & 5,2 & 3,8 & $6,490 \mathrm{E}+24$ \\
\hline 49 & $29 / 01 / 2010$ & $17: 52: 23$ & 4,83 & 76,07 & 133,4 & 5,0 & 4,9 & 3,7 & $3,300 \mathrm{E}+24$ \\
\hline
\end{tabular}


En la TABLA 1, se muestra la fecha y tiempo de ocurrencia de cada uno de los eventos, su localización hipocentral (epicentro en coordenadas geográficas y profundidad en kilómetros), magnitud reportadas en las escalas Mw, mb y Ms y el escalar de momento sísmico en dinas por centímento.

En la FIGURA 4 se muestra en un corte oeste-este, la distribución en profundidad de los sismos cuyos mecanismos focales son analizados en este trabajo.
La localización hipocentral de los eventos señala que su profundidad se distribuye entre superficiales e intermedios, hasta $215 \mathrm{~km}$ aproximadamente, asociados a la subducción de la placa Nazca, y unos pocos eventos superficiales que corresponden a la actividad de fuentes corticales en la placa continental sudamericana. En la FIGURA 5 se presenta la solución gráfica del mecanismo focal (beach ball) y ubicación geográfica de cada uno de los terremotos considerados, la numeración de los eventos es correspondiente con la TABLA 1.

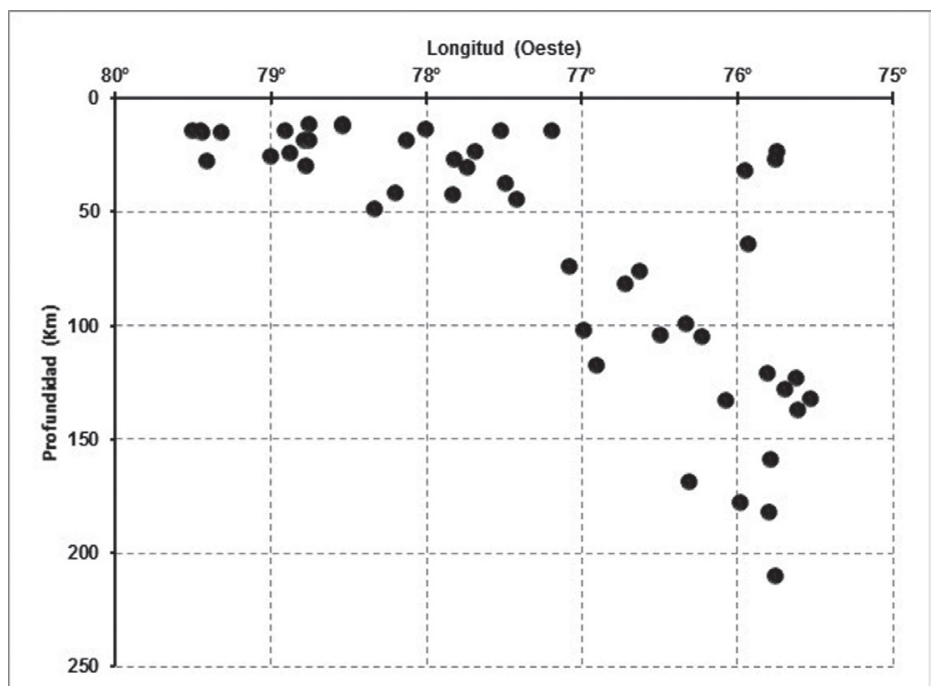

FIGURA 4. Distribución en profundidad de los sismos $M w \geq 4,8$ con solución del mecanismo focal en la región del Valle del Cauca y zonas aledañas, ocurridos en el periodo $1978-2010$.

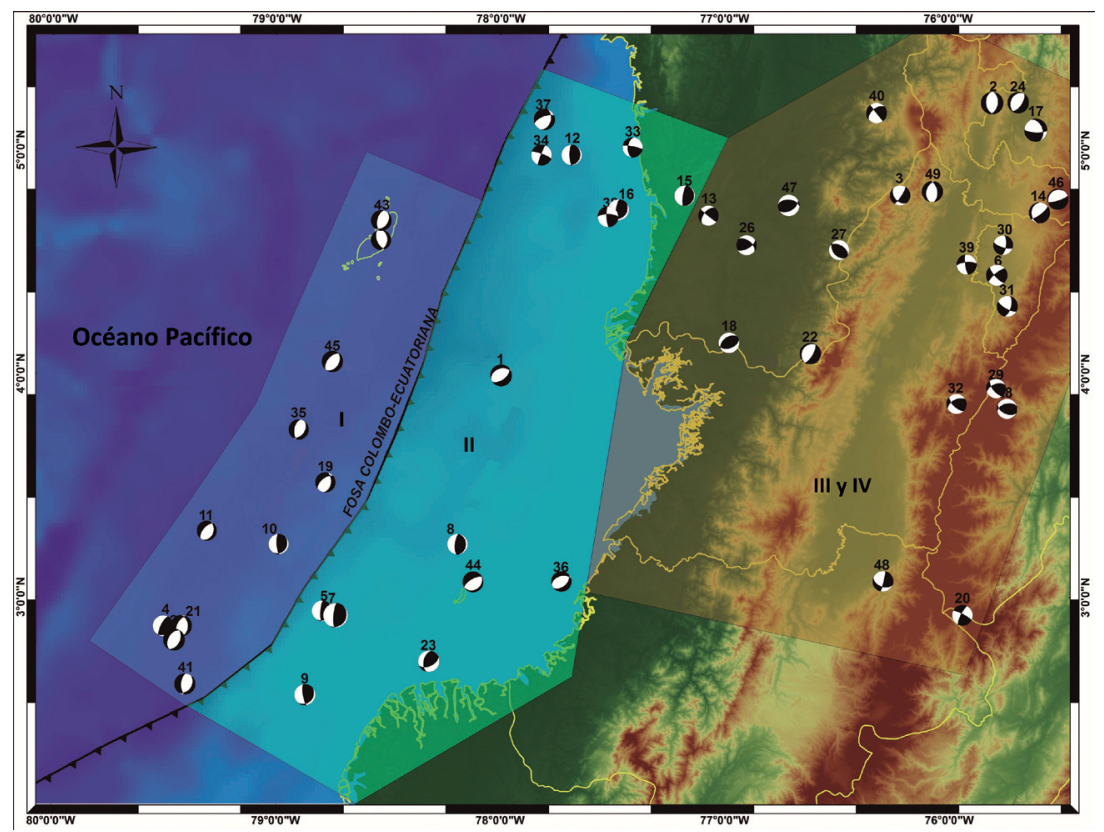

FIGURA 5. Representación gráfica del mecanismo focal de los sismos con magnitud $M w \geq 4,8$ ocurridos en la región del Valle del Cauca y zonas aledañas en el periodo 1978 - 2010 y su agrupación en ambientes sismotectónicos delimitados por los polígonos sombreados. Los ambientes III y IV son delimitados por el mismo polígono, diferenciándose en la profundidad de los eventos sísmicos (Fuente: Catálogos del ISC y CMT. 
La solución del mecanismo focal de los terremotos considera dos planos nodales descritos por los ángulos: azimut o rumbo $(\varphi)$, buzamiento $(\delta)$ y deslizamiento $(\lambda)$ y los ejes principales de esfuerzos $T$ (Tensión) y $P$ (Presión), orientados mediante los ángulos $\Theta$ (ángulo que forma el eje con la vertical o plunge) y $\phi$ (con el norte o azimut) (Buforn, 1994). La selección del plano de falla para cada evento se hizo a partir de la información de la geología estructural de la región, en especial de los mapas de fallas activas (París et al., 1992; París et al., 2000; Montes y Sandoval, 2001; Nivia, 2001), luego se realizó la representación gráfica del mecanismo focal de acuerdo a los ángulos del plano de falla seleccionado. La herramienta usada para este propósito es "Focal Mechanisms" que se encuentra en línea en la página de George Helffrich (http://www1.gly.bris.ac.uk/ george/ focmec.html) en la cual solo es necesario ingresar los ángulos de la orientación del plano. La interpretación del tipo de solución del plano de falla se hizo a partir del ángulo de deslizamiento (rake of slip) acorde con los criterios establecidos por Cronin (2010).
Considerando las características de la sismicidad y la distribución de las soluciones de los mecanismos focales encontrados en la región de estudio y acorde con Lay et al. (1989) quien define ambientes sismotectónicos de la placa en subducción (antes, durante y después), en el presente trabajo de definieron cuatro ambientes sismotectónicos, que se describen a continuación.

\section{CARATERIZACIÓN DE AMBIENTES SISMOTECTÓNICOS}

Como se mencionó, de acuerdo con las características sismotectónicas en la región de estudio se han definido cuatro ambientes tectónicos denominados: Ambiente I - Zona antes de la fosa, Ambiente II - Zona de flexión e interacción interplacas, Ambiente III - Zona continental superficial y Ambiente IV - Zona de Benioff intermedia (FIGURA 6). Cada uno de ellos se describe a continuación.

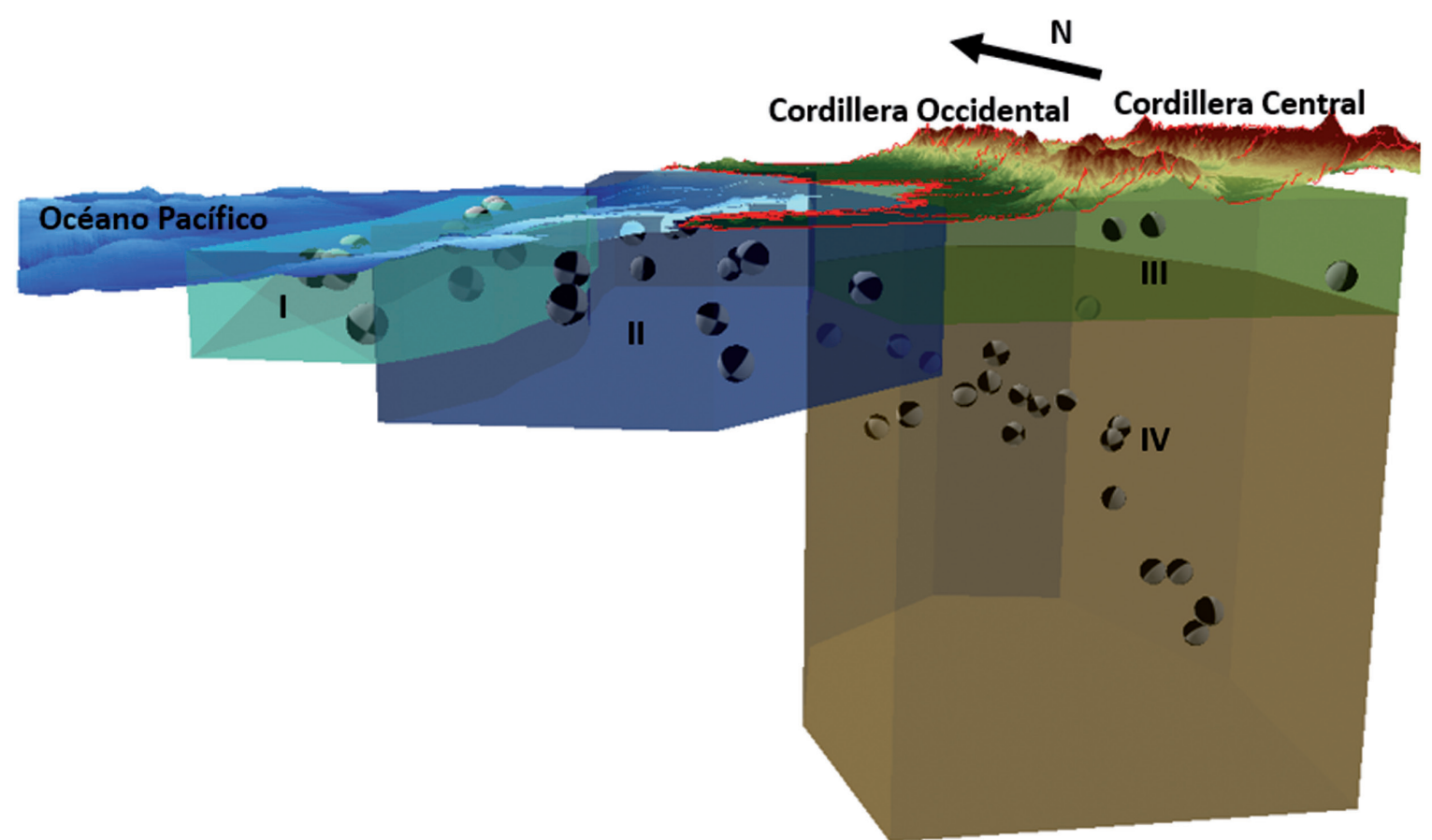

FIGURA 6. Esquema tridimensional de los mecanismos focales de los terremotos para los cuatro ambientes tectónicos determinados.

\section{Ambiente I - Zona antes de la fosa}

El "ambiente sismotectónico I" está determinado por la sismicidad que se presenta en la zona antes de la fosa Colombo-Ecuatoriana, donde ocurren eventos superficiales. En este caso, se han escogido los eventos localizados entre las coordenadas $2,51^{\circ}$ y $4,99^{\circ}$ de latitud norte y $79.82^{\circ}$ y $78.08^{\circ}$ de longitud oeste. La profundidad de estos sismos oscila entre 12 y $28,6 \mathrm{~km}$. De esta manera, se seleccionaron las soluciones de mecanismos focales de 11 eventos sísmicos (TABLA 2 y FIGURA 6). 
TABLA 2. Solución del mecanismo focal de sismos del Ambiente tectónico I (zona antes de la Fosa) dentro de la región del Valle del Cauca y zonas aledañas, ocurridos en el periodo entre $1978-2010$.

\begin{tabular}{|c|c|c|c|c|c|c|c|c|c|c|c|c|c|}
\hline \multirow{3}{*}{ No. } & \multirow{3}{*}{$\begin{array}{c}\text { Fecha } \\
\text { dd:mm:aa }\end{array}$} & \multirow{2}{*}{\multicolumn{3}{|c|}{ Localización }} & \multirow{2}{*}{\multicolumn{3}{|c|}{ Plano de falla }} & \multicolumn{4}{|c|}{ Eje de esfuerzo } & \multirow{3}{*}{$\begin{array}{l}\text { Tipo de } \\
\text { Mecanismo }\end{array}$} & \multirow{3}{*}{ Grafico* } \\
\hline & & & & & & & & \multicolumn{2}{|c|}{$\mathbf{P}$} & \multicolumn{2}{|c|}{$\mathbf{T}$} & & \\
\hline & & Lat $\left({ }^{\circ} \mathbf{N}\right)$ & Long $\left({ }^{\circ} \mathrm{W}\right)$ & Prof. $(\mathrm{Km})$ & $\varphi$ & $\delta$ & $\lambda$ & $(\phi)$ & $(\Theta)$ & $(\phi)$ & $(\Theta)$ & & \\
\hline 4 & $13 / 12 / 1979$ & 2,88 & 79,49 & 15,0 & 207 & 72 & 84 & 302 & 27 & 108 & 63 & Inverso & \\
\hline 10 & $10 / 06 / 1985$ & 3,24 & 78,99 & 26,0 & 176 & 74 & 79 & 275 & 29 & 70 & 59 & Inverso & \\
\hline 11 & $25 / 01 / 1987$ & 3,30 & 79,31 & 15,4 & 221 & 60 & -79 & 159 & 73 & 303 & 14 & Normal oblicuo & \\
\hline 19 & 03/06/1994 & 3,52 & 78,78 & 19,0 & 10 & 42 & -123 & 196 & 67 & 303 & 7 & Normal oblicuo & \\
\hline 21 & 26/11/1994 & 2,87 & 79,43 & 15,9 & 206 & 57 & -73 & 159 & 72 & 284 & 10 & Normal & \\
\hline 25 & $13 / 11 / 1995$ & 2,83 & 79,44 & 15,0 & 28 & 45 & -90 & 180 & 90 & 118 & 0 & Normal & \\
\hline 35 & $21 / 12 / 2002$ & 3,76 & 78,90 & 15,0 & 36 & 40 & -70 & 50 & 76 & 292 & 7 & Normal & \\
\hline 41 & 03/08/2005 & 2,62 & 79,40 & 28,6 & 34 & 35 & -64 & 54 & 70 & 285 & 13 & Normal & \\
\hline 42 & $17 / 03 / 2007$ & 4,61 & 78,53 & 13,1 & 55 & 37 & -67 & 72 & 73 & 308 & 10 & Normal & \\
\hline 43 & $18 / 03 / 2007$ & 4,69 & 78,53 & 12,0 & 38 & 36 & -69 & 64 & 74 & 294 & 10 & Normal & \\
\hline 45 & $23 / 02 / 2008$ & 4,06 & 78,75 & 12,0 & 31 & 38 & -98 & 161 & 81 & 307 & 7 & Normal & \\
\hline
\end{tabular}

* - el gráfico corresponde a la proyección en profundidad de los ejes de esfuerzos P y T y la solución del mecanismo, la elipse de color representa la orientación del plano de falla. Los gráficos fueron hechos en el programa "3D Focal Mechanisms" de Keith Labay y Peter Haessler (http://pubs.usgs.gov/ds/2007/241).

En la TABLA 2 se presentan los parámetros de la solución de los mecanismos focales de los eventos asociados con el ambiente sismotectónico I, acompañado de un gráfico de la proyección del mecanismo en profundidad, el cual a su vez indica la orientación de los ejes de esfuerzo $P$ y $T$ y del plano de falla. La numeración de cada evento corresponde al número asignado a cada mecanismo focal en la TABLA 1 y la FIGURA 6.

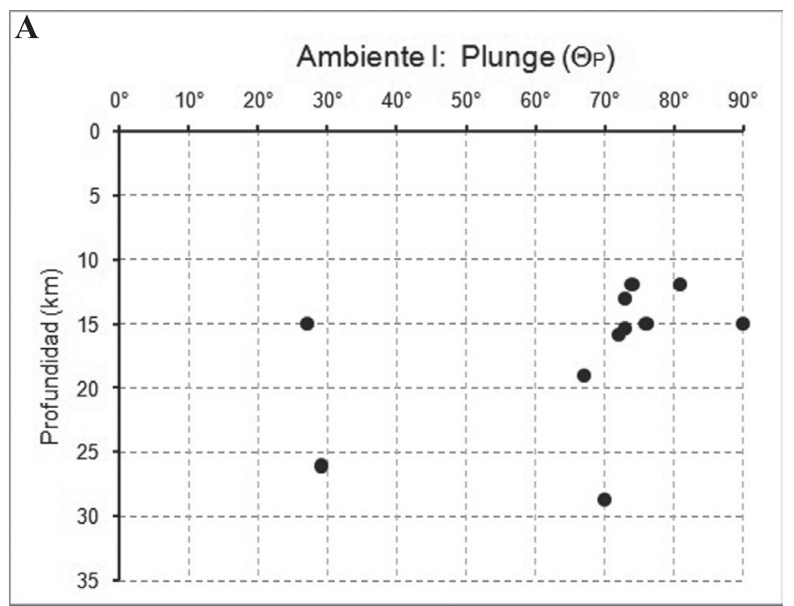

Se aprecia que la mayoría de estos eventos son de tipo extensivo, respondiendo a las fuerzas generadas por el proceso de expansión que se produce en la zona de dorsal interoceánica. La distribución del ángulo de inmersión de los ejes de esfuerzos compresivo $(P)$ y de tensión $(T)$ de cada uno de los mecanismos focales para cada sismo considerado en este ambiente sismotectónico, muestra que el eje de esfuerzo compresivo en la mayoría de éstos mantiene un ángulo de inmersión casi horizontal entre $70^{\circ}$ y $90^{\circ}$, mientras que el ángulo del eje de esfuerzo de tensión está casi vertical entre $0^{\circ}$ y $15^{\circ}$ (FIGURA 7).

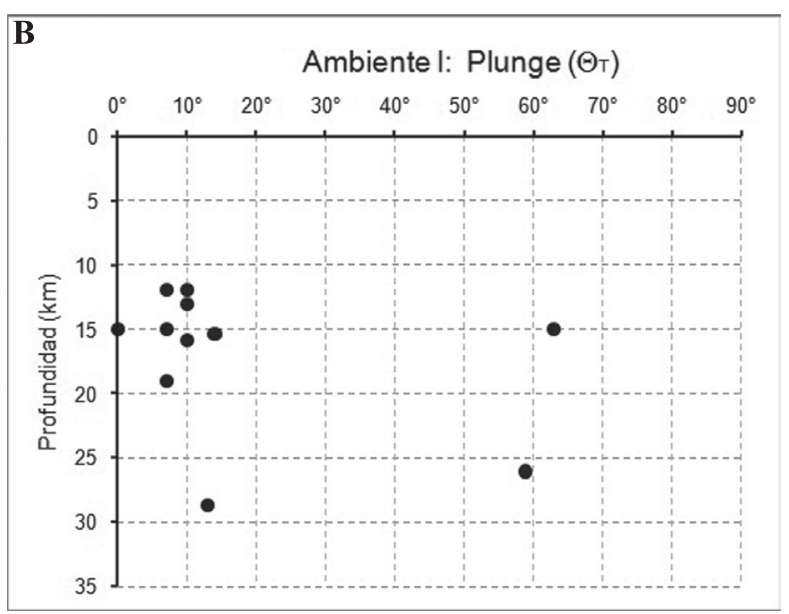

FIGURA 7. Representación de los ángulos de inmersión (Plunge) de los ejes de esfuerzos, A. compresivo $(P)$ y B. de tensión $(T)$ en el ambiente sismotectónico I, zona antes de la fosa. 


\section{Ambiente II - Zona de flexión e interacción interplacas}

Este ambiente se asocia con los procesos que generan los sismos en la zonas de flexión y de interacción interplacas, es determinado entre las coordenadas $2,08^{\circ}$ y $5,37^{\circ}$ de latitud norte y $79.41^{\circ}$ y $77.88^{\circ}$ de longitud oeste. La profundidad de los sismos está entre 14 y 49,4 $\mathrm{km}$. Así, se reunieron los mecanismos focales de 15 eventos. Por su naturaleza, los sismos de este ambiente son de mayor magnitud que los del ambiente anterior.

Los parámetros de la solución de los mecanismos focales de los eventos asociados con el ambiente sismotectónico II, se muestran en la TABLA 3, donde también se presenta el gráfico de la proyección del mecanismo en profundidad, indicándose la orientación de los ejes de esfuerzo $P$ y $T$ y el respectivo plano de falla. Puede observarse la presencia de eventos tanto de tipo normal como inverso.

TABLA 3. Solución del mecanismo focal de sismos del Ambiente tectónico II (zonas de flexión e interacción interplacas) dentro de la región del Valle del Cauca y zonas aledañas, ocurridos en el periodo entre 1978 - 2010.

\begin{tabular}{|c|c|c|c|c|c|c|c|c|c|c|c|c|c|}
\hline \multirow{3}{*}{ No. } & \multirow{3}{*}{$\begin{array}{c}\text { Fecha } \\
\text { dd:mm:aa }\end{array}$} & \multirow{2}{*}{\multicolumn{3}{|c|}{ Localización }} & \multirow{2}{*}{\multicolumn{3}{|c|}{ Plano de falla }} & \multicolumn{4}{|c|}{ Eje de esfuerzo } & \multirow{3}{*}{$\begin{array}{c}\text { Tipo de } \\
\text { Mecanismo }\end{array}$} & \multirow{3}{*}{ Grafico* } \\
\hline & & & & & & & & \multicolumn{2}{|c|}{$\mathbf{P}$} & \multicolumn{2}{|c|}{$\mathbf{T}$} & & \\
\hline & & $\begin{array}{l}\text { Lat } \\
\left({ }^{\circ} \mathrm{N}\right)\end{array}$ & $\begin{array}{l}\text { Long } \\
\left({ }^{\circ} \mathrm{W}\right)\end{array}$ & $\begin{array}{l}\text { Prof. } \\
\text { (Km) }\end{array}$ & $\varphi$ & $\delta$ & $\lambda$ & $(\phi)$ & $(\Theta)$ & $(\phi)$ & $(\Theta)$ & & \\
\hline 1 & $16 / 02 / 1978$ & 4,00 & 78,00 & 14,0 & 53 & 55 & -100 & 289 & 78 & 150 & 9 & Normal oblicuo & \\
\hline 5 & 07/01/1980 & 2,94 & 78,77 & 30,8 & 185 & 77 & 84 & 280 & 31 & 87 & 58 & Normal oblicuo & \\
\hline 7 & 01/07/1980 & 2,93 & 78,75 & 19,0 & 185 & 77 & 84 & 280 & 31 & 87 & 58 & Inverso & \\
\hline 8 & 03/09/1980 & 3,24 & 78,19 & 42,1 & 186 & 70 & 92 & 275 & 25 & 99 & 65 & Inverso & \\
\hline 9 & 08/01/1982 & 2,57 & 78,87 & 25,1 & 171 & 76 & 73 & 247 & 29 & 59 & 56 & Inverso & \\
\hline 12 & 20/09/1988 & 4,99 & 77,68 & 24,1 & 179 & 73 & 85 & 272 & 27 & 82 & 62 & Inverso & \\
\hline 15 & 19/11/1991 & 4,80 & 77,18 & 14,9 & 188 & 77 & 89 & 279 & 32 & 97 & 58 & Inverso & \\
\hline 16 & 10/12/1991 & 4,74 & 77,48 & 38,4 & 186 & 72 & 76 & 287 & 26 & 76 & 60 & Inverso & \\
\hline 23 & 04/12/1995 & 2,72 & 78,32 & 49,4 & 65 & 53 & 127 & 129 & 2 & 36 & 61 & Inverso oblicuo & \\
\hline 33 & $02 / 07 / 2002$ & 5,02 & 77,41 & 45,3 & 185 & 51 & 170 & 46 & 21 & 151 & 33 & Desgarre sinestral & \\
\hline 34 & 08/08/2002 & 4,98 & 77,82 & 43,3 & 200 & 85 & 170 & 246 & 3 & 155 & 11 & Desgarre sinestral & \\
\hline 36 & $22 / 08 / 2003$ & 3,07 & 77,73 & 31,4 & 50 & 47 & -106 & 246 & 78 & 152 & 1 & Normal oblicuo & \\
\hline 37 & $05 / 11 / 2003$ & 5,14 & 77,81 & 27,6 & 15 & 38 & -137 & 201 & 59 & 317 & 15 & Normal oblicuo & \\
\hline 38 & $15 / 11 / 2004$ & 4,70 & 77,51 & 15,0 & 177 & 79 & 85 & 271 & 34 & 81 & 55 & Inverso & \\
\hline 44 & $10 / 09 / 2007$ & 3,08 & 78,12 & 18,9 & 54 & 23 & -95 & 153 & 68 & 328 & 22 & Normal & \\
\hline
\end{tabular}

* - el gráfico corresponde a la proyección en profundidad de los ejes de esfuerzos P y T y la solución del mecanismo.

En la FIGURA 8 se representa gráficamente la distribución en profundidad del ángulo de inmersión de los ejes de esfuerzos compresivo $(P)$ y distensivo $(T)$ de los mecanismos focales para los eventos considerados en este ambiente. Esta figura muestra que el eje de esfuerzo compresivo mantiene un ángulo de inmersión casi oblicuo entre $20^{\circ}$ y $35^{\circ}$. También se nota que en algunos eventos, especialmente los de tipo normal, el ángulo de inmersión del eje de esfuerzo compresivo tiende a la horizontalidad con valores cercanos a los $80^{\circ}$, mientras que para el caso del eje de esfuerzo de tensión se observa que en la mayoría de los casos el ángulo de inmersión en promedio es de $60^{\circ}$, oscilando entre $55^{\circ}$ y $65^{\circ}$. 

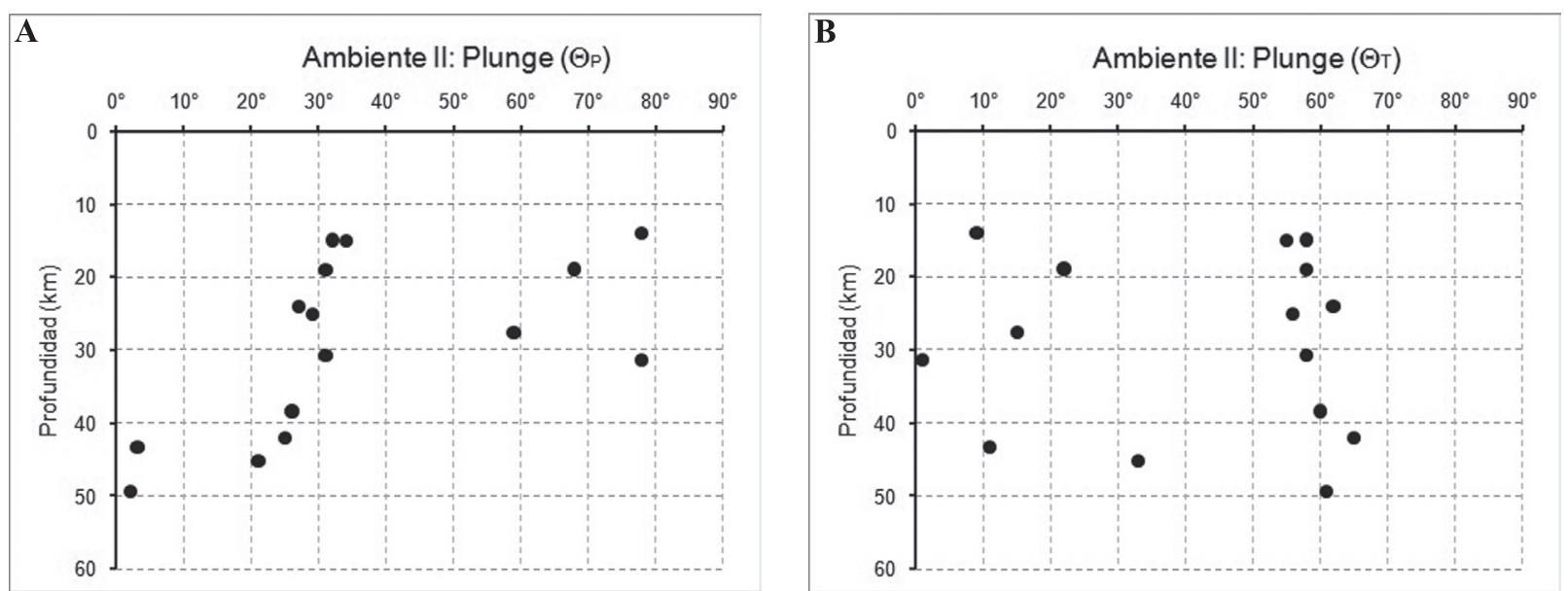

FIGURA 8. Representación de los ángulos de inmersión (Plunge) de los ejes de esfuerzos, A. Compresivo (P) y B. Tensión (T) en el Ambiente Sismotectónico II, zona de flexión e interacción interplacas.

Los datos obtenidos son concordantes con lo propuesto por Christensen and Ruff (1988), al demostrar que los terremotos que ocurren en este tipo de ambiente son eventos normales o inversos con ejes de tracción y de compresión orientados aproximadamente subhorizontalmente y perpendiculares al eje de la fosa. La distribución espacial de los eventos de tipo normal y de compresión dentro de la litosfera subducida responden a la flexión de la placa, siendo explicados por modelos que establecen esfuerzos de tensión en la litosfera superior y una región de compresión más profunda (Chapple and Forsyth, 1979; Seno and Yamanaka, 1996).

\section{Ambiente III - Zona continental superficial}

El ambiente tectónico III corresponde a la sismicidad superficial en la placa continental, definida entre las coordenadas $2,66^{\circ}$ y $5,59^{\circ}$ de latitud norte y $77.44^{\circ}$ y $75.46^{\circ}$ de longitud oeste, y la profundidad de los sismos entre 24,3 y $32,6 \mathrm{~km}$. De esta manera, para este ambiente se reunieron los mecanismos focales de tres eventos sísmicos (TABLA 4).

En la TABLA 4 se presenta la solución del mecanismo focal de cada uno de los eventos asociados a este ambiente sismotectónico. También se muestra el gráfico de la proyección del mecanismo en profundidad, que a su vez indica la orientación de los ejes de esfuerzo compresivo $(P)$ y distensivo $(T)$ y el plano de falla. Debido al carácter cortical de estos eventos, su ocurrencia se asocia al sistema de fallamientos existentes en la región, entre los cuales se encuentran fallas tanto de tipo normal como inverso (Murcia, 1981; Page, 1986; París et al., 1992; París et al., 2000; Nivia, 2001; INGEOMINAS, 2005; López, 2006).
En este caso, la distribución en profundidad del ángulo de inmersión de los ejes de esfuerzos compresivo $(P)$ y distensivo $(T)$ de los mecanismos focales de los eventos considerados, permite establecer que el ángulo de inmersión del eje $\mathrm{P}$, aunque no hay uniformidad en la baja cantidad de eventos, está por debajo de $35^{\circ}$. Mientras que la inmersión del eje de esfuerzo $\mathrm{T}$ es menor de $20^{\circ}$ (FIGURA 9).

\section{Ambiente IV - Zona de Benioff intermedia}

En el presente estudio se reconoce la zona de Benioff intermedia como el ambiente sismotectónico IV, definida entre las coordenadas $2,66^{\circ}$ y $5,59^{\circ}$ de latitud norte y $77.44^{\circ}$ y $75.46^{\circ}$ de longitud oeste, y profundidad de los sismos entre 64,5 y $211 \mathrm{~km}$.

Las soluciones de los mecanismos focales de los eventos asociados con el ambiente sismotectónico IV se muestran en la TABLA 5, que a su vez presenta el gráfico de la proyección del mecanismo en profundidad, indicándose la orientación de los ejes de esfuerzo $P$ y $T$ y el plano de falla. En este ambiente no se puede establecer un tipo de mecanismo focal predominante, lo que señala el carácter complejo de los procesos tectónicos que ocurren a esta profundidad. La distribución en profundidad del ángulo de inmersión de los ejes de esfuerzos compresivo $(P)$ y distensivo $(T)$ de los mecanismos focales para los eventos considerados en este ambiente se puede apreciar que el ángulo de inmersión tanto para el eje de esfuerzo compresivo como para el eje de esfuerzo distensivo, no guarda un patrón de orientación específico (FIGURA 10), lo que se puede atribuir a la variabilidad y complejidad de los procesos tectónicos que a esta profundidad se generan debido a la alta temperatura que causa la variabilidad de los tipos de esfuerzos. 
En la TABLA 6 se presentan un resumen las principales características de los ambientes sismotectónicos definidos.

TABLA 4. Solución del mecanismo focal de sismos del Ambiente tectónico III (zonas de Benioff intermedia) dentro de la región del Valle del Cauca y zonas aledañas, ocurridos en el periodo entre $1978-2010$.

\begin{tabular}{|c|c|c|c|c|c|c|c|c|c|c|c|c|c|}
\hline \multirow{3}{*}{ No. } & \multirow{3}{*}{$\begin{array}{c}\text { Fecha } \\
\text { dd:mm:aa }\end{array}$} & \multirow{2}{*}{\multicolumn{3}{|c|}{ Localización }} & \multirow{2}{*}{\multicolumn{3}{|c|}{ Plano de falla }} & \multicolumn{4}{|c|}{ Eje de esfuerzo } & \multirow{3}{*}{ Tipo de Mecanismo } & \multirow{3}{*}{ Grafico* } \\
\hline & & & & & & & & \multicolumn{2}{|c|}{$\mathbf{P}$} & \multicolumn{2}{|c|}{$\mathbf{T}$} & & \\
\hline & & $\begin{array}{l}\text { Lat } \\
\left({ }^{\circ} \mathbf{N}\right)\end{array}$ & $\begin{array}{l}\text { Long } \\
\left({ }^{\circ} \mathrm{W}\right)\end{array}$ & $\begin{array}{l}\text { Prof. } \\
(\mathrm{Km})\end{array}$ & $\varphi$ & $\delta$ & $\lambda$ & $(\phi)$ & $(\Theta)$ & $(\phi)$ & $(\Theta)$ & & \\
\hline 2 & $29 / 05 / 1979$ & 5,22 & 75,80 & 122,0 & 174 & 44 & -100 & 350 & 83 & 91 & 1 & Normal & \\
\hline 3 & $23 / 11 / 1979$ & 4,81 & 76,22 & 105,4 & 137 & 41 & -163 & 342 & 42 & 95 & 24 & Normal con desgarre dextral & \\
\hline 6 & $25 / 06 / 1980$ & 4,44 & 75,78 & 159,5 & 137 & 76 & 164 & 184 & 1 & 94 & 21 & Desgarre dextral & \\
\hline 13 & $29 / 11 / 1988$ & 4,72 & 77,07 & 75,0 & 220 & 51 & 3 & 180 & 24 & 76 & 28 & Desgarre sinestral & \\
\hline 14 & $23 / 11 / 1990$ & 4,73 & 75,60 & 138,0 & 53 & 75 & -72 & 129 & 28 & 346 & 56 & Normal oblicuo & \\
\hline 17 & $15 / 08 / 1992$ & 5,11 & 75,61 & 124,0 & 340 & 40 & -28 & 322 & 49 & 208 & 19 & Normal con desgarre dextral & \\
\hline 18 & $12 / 10 / 1993$ & 4,15 & 76,98 & 102,9 & 234 & 44 & 77 & 154 & 2 & 53 & 81 & Inverso oblicuo & \\
\hline 22 & $08 / 02 / 1995$ & 4,10 & 76,62 & 77,1 & 30 & 69 & -90 & 300 & 66 & 120 & 24 & Normal oblicuo & \\
\hline 24 & $19 / 08 / 1995$ & 5,22 & 75,69 & 128,7 & 34 & 61 & -85 & 318 & 74 & 120 & 16 & Normal oblicuo & \\
\hline 26 & $11 / 09 / 1996$ & 4,59 & 76,90 & 118,2 & 61 & 63 & 45 & 181 & 7 & 280 & 50 & Inverso con desgarre dextral & \\
\hline 27 & $19 / 02 / 1997$ & 4,56 & 76,49 & 105,0 & 299 & 50 & 77 & 38 & 4 & 150 & 80 & Inverso oblicuo & \\
\hline 28 & 02/09/1997 & 3,85 & 75,75 & 211,0 & 256 & 48 & 62 & 186 & 0 & 95 & 70 & Inverso & \\
\hline 29 & $11 / 12 / 1997$ & 3,93 & 75,79 & 183,0 & 140 & 66 & 139 & 197 & 8 & 99 & 46 & Inverso con desgarre dextral & \\
\hline 32 & $22 / 09 / 2001$ & 3,87 & 75,97 & 178,6 & 133 & 65 & 139 & 195 & 7 & 97 & 46 & Inverso con desgarre sinestral & \\
\hline 39 & $08 / 03 / 2005$ & 4,50 & 75,92 & 64,5 & 171 & 79 & 159 & 219 & 6 & 127 & 22 & Desgarre sinestral & \\
\hline 40 & $21 / 04 / 2005$ & 5,18 & 76,32 & 99,7 & 232 & 56 & 1 & 192 & 23 & 91 & 24 & Desgarre sinestral & \\
\hline 46 & $13 / 09 / 2008$ & 4,79 & 75,52 & 132,9 & 72 & 79 & -85 & 349 & 56 & 158 & 33 & Normal oblicuo & \\
\hline 47 & 08/09/2009 & 4,77 & 76,71 & 82,7 & 60 & 49 & 66 & 166 & 2 & 261 & 72 & Inverso oblicuo & \\
\hline 48 & $18 / 12 / 2009$ & 3,08 & 76,30 & 169,2 & 14 & 84 & -44 & 322 & 34 & 70 & 25 & Normal con desgarre sinestral & \\
\hline 49 & $29 / 01 / 2010$ & 4,83 & 76,07 & 133,4 & 352 & 51 & -105 & 206 & 77 & 93 & 5 & Normal & \\
\hline
\end{tabular}

* - el gráfico corresponde a la proyección en profundidad de los ejes de esfuerzos P y T y la solución del mecanismo.
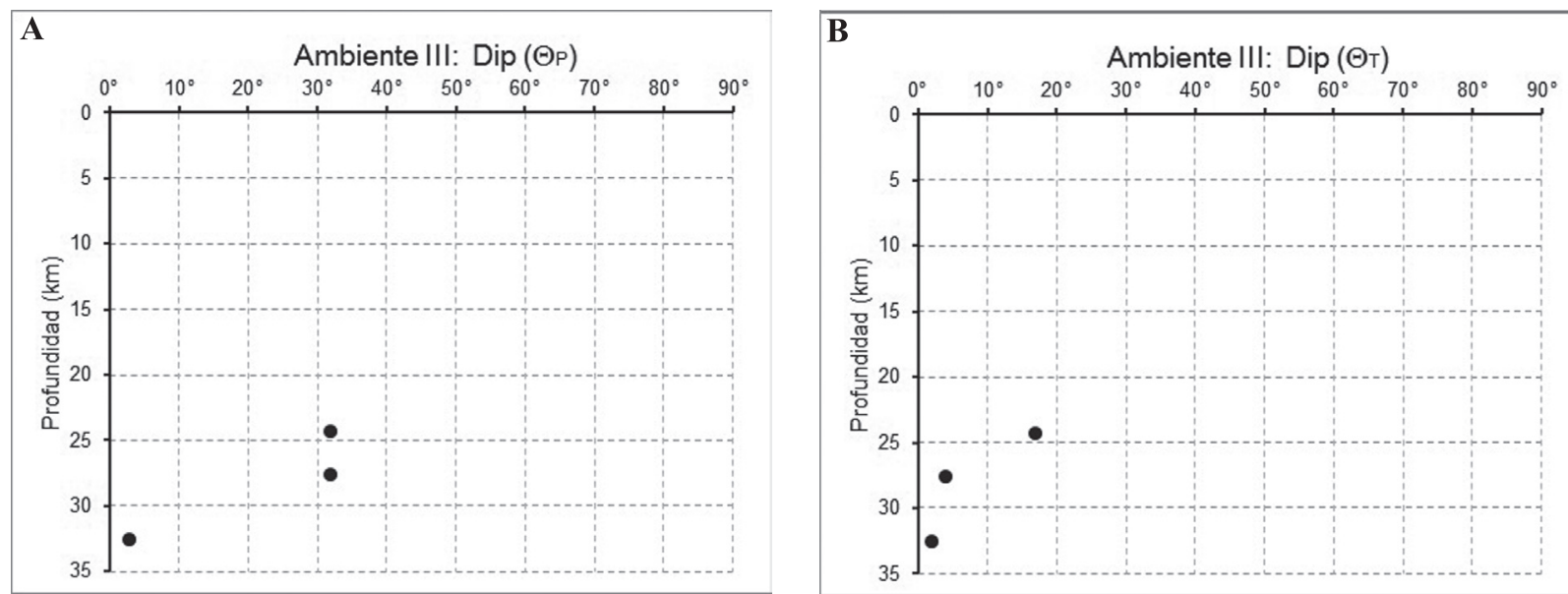

FIGURA 9. Representación de los ángulos de inmersión (Plunge) de los ejes de esfuerzos, A. Compresivo $(P)$ y B. Tensión $(T)$ en el Ambiente Sismotectónico III, zona continental superficial. 
TABLA 5. Solución del mecanismo focal de sismos del Ambiente tectónico IV (zona continental superficial) dentro de la región del Valle del Cauca y zonas aledañas, ocurridos en el periodo entre $1978-2010$.

\begin{tabular}{|c|c|c|c|c|c|c|c|c|c|c|c|c|c|}
\hline \multirow{3}{*}{ No. } & \multirow{3}{*}{$\begin{array}{c}\text { Fecha } \\
\text { dd:mm:aa }\end{array}$} & \multirow{2}{*}{\multicolumn{3}{|c|}{ Localización }} & \multirow{2}{*}{\multicolumn{3}{|c|}{ Plano de falla }} & \multicolumn{4}{|c|}{ Eje de esfuerzo } & \multirow{3}{*}{ Tipo de Mecanismo } & \multirow{3}{*}{ Grafico* } \\
\hline & & & & & & & & \multicolumn{2}{|c|}{$\mathbf{P}$} & \multicolumn{2}{|c|}{$\mathbf{T}$} & & \\
\hline & & $\begin{array}{l}\text { Lat } \\
\left({ }^{\circ} \mathbf{N}\right)\end{array}$ & $\begin{array}{l}\text { Long } \\
\left({ }^{\circ} \mathrm{W}\right)\end{array}$ & $\begin{array}{l}\text { Prof. } \\
(\mathbf{K m})\end{array}$ & $\varphi$ & $\delta$ & $\lambda$ & $(\phi)$ & $(\Theta)$ & $(\phi)$ & $(\Theta)$ & & \\
\hline 20 & 06/06/1994 & 2,93 & 75,94 & 32,6 & 206 & 76 & 170 & 72 & 3 & 163 & 17 & $\begin{array}{c}\text { Inverso con Desgarre } \\
\text { dextral }\end{array}$ & \\
\hline 30 & 25/01/1999 & 4,58 & 75,75 & 27,7 & 8 & 65 & -21 & 329 & 32 & 236 & 4 & $\begin{array}{c}\text { Normal con desgarre } \\
\text { sinestral }\end{array}$ & \\
\hline 31 & 25/01/1999 & 4,31 & 75,74 & 24,3 & 17 & 67 & -23 & 337 & 32 & 246 & 2 & $\begin{array}{c}\text { Normal con desgarre } \\
\text { sinestral }\end{array}$ & \\
\hline
\end{tabular}

* - el gráfico corresponde a la proyección en profundidad de los ejes de esfuerzos P y T y la solución del mecanismo.
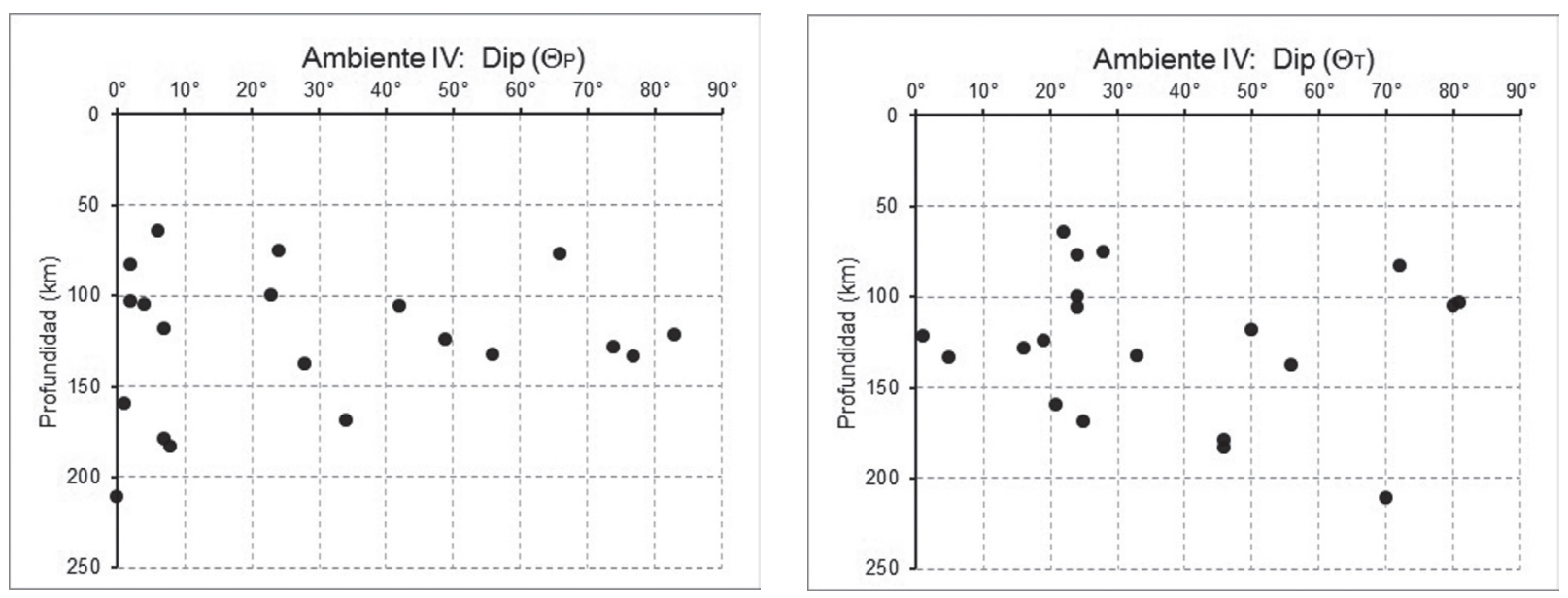

FIGURA 10. Representación de los ángulos de inmersión (Plunge) de los ejes de esfuerzos, A. Compresivo $(P)$ y B. Tensión $(T)$ en el Ambiente Sismotectónico IV, zona de Benioff intermedia.

TABLA 6. Resumen de las características de los Ambiente Sismotectónicos definidos en este estudio.

\begin{tabular}{|c|c|c|c|c|}
\hline Ambiente Sismotectónico & Relación M0 vs Mw & $\begin{array}{l}\text { Mecanismo } \\
\text { dominante }\end{array}$ & $\begin{array}{c}\text { Dominio ángulo de } \\
\text { Inmersión } \\
\text { Eje } P \text { (grados) } \\
\end{array}$ & $\begin{array}{c}\text { Dominio ángulo de } \\
\text { Inmersión } \\
\text { Eje } T \text { (grados) } \\
\end{array}$ \\
\hline $\begin{array}{l}\text { Ambiente I: } \\
\text { Zona antes de la fosa }\end{array}$ & $\begin{array}{c}\log M_{0}=0,63 M w+24,03 \\
\mathrm{R}^{2}=0,95\end{array}$ & Normal & $70-90$ & $0-15$ \\
\hline $\begin{array}{l}\text { Ambiente II: } \\
\text { Zona de flexión e interacción } \\
\text { interplacas }\end{array}$ & $\begin{array}{c}\log M_{0}=0,37 M w+25,49 \\
\mathrm{R}^{2}=0,64\end{array}$ & Inverso & $20-35$ & $55-65$ \\
\hline $\begin{array}{l}\text { Ambiente III: } \\
\text { Zona de Benioff intermedia }\end{array}$ & $\begin{array}{c}\log M_{0}=0,41 M w+25,24 \\
\mathrm{R}^{2}=0,91\end{array}$ & Normal & No distinguido & No distinguido \\
\hline $\begin{array}{l}\text { Ambiente IV: } \\
\text { Zona continental superficial }\end{array}$ & Datos insuficientes & $\begin{array}{c}\text { Normal, pocos } \\
\text { datos }\end{array}$ & $\begin{array}{c}<35 \\
\text { pocos datos }\end{array}$ & $\begin{array}{c}<20 \\
\text { pocos datos }\end{array}$ \\
\hline
\end{tabular}

\section{DISCUSIÓN Y CONCLUSIONES}

\section{Implicaciones Sismotectónicas}

A partir de la orientación de los ejes de esfuerzos principales $P$ y $T$ se realiza el mapa sismotectónico simplificado (FIGURA 11), que muestra que en la zona antes de la fosa Colombo-Ecuatoriana predomina un régimen de esfuerzos distensivo. Los terremotos que ocurren esta zona se deben, por un lado, a la fuerza de empuje desde la dorsal meso-oceánica y, por el otro, a la fuerza resistente que aparece por el choque con la placa continental. La primera de ellas es la responsable del movimiento de la placa oceánica, que en este caso se mueve con velocidad de entre 5 a $8 \mathrm{~cm} /$ año (Pennington, 1981; Kellogg et al., 1989; Freymueller et al., 1993; 
Gutscher et al., 1999; Trenkamp et al., 2002, Trenkamp et al., 2004). Mientras que la segunda fuerza hace que en la fosa se produzca una alta velocidad de retroceso de la placa oceánica (Capitanio et al., 2007).

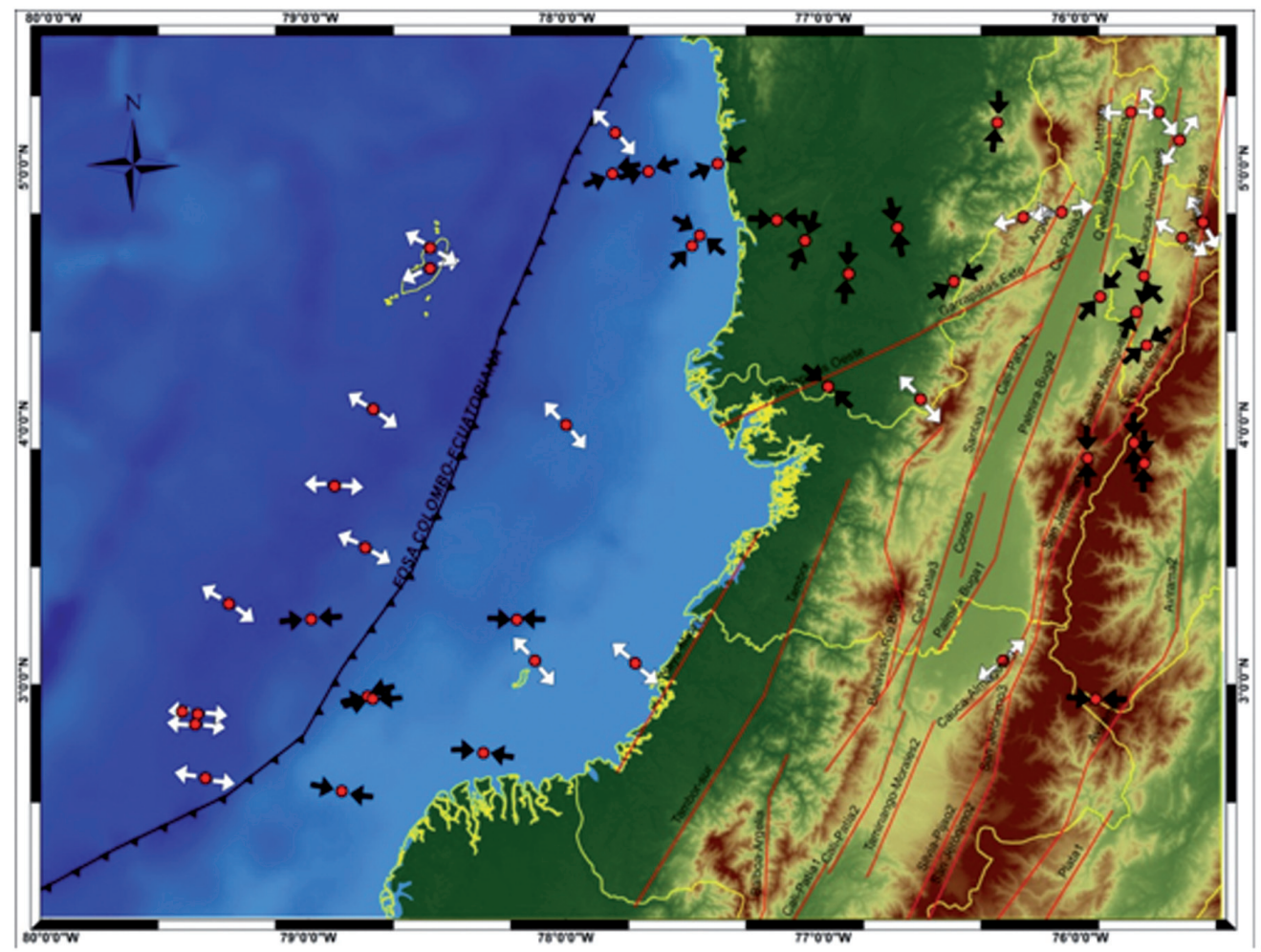

FIGURA 11. Mapa de esfuerzos sismotectónicos en la región del Valle del Cauca y zonas aledañas, las flechas de color negro representan esfuerzos compresivos y las fechas de color blanco representan esfuerzos de tensión.

Los sismos que ocurren en la zona de contacto entre placas oceánica y continental son superficiales y predominantemente de carácter compresivo como resultado esencialmente del encuentro de las fuerzas de arrastre del manto o fuerza de empuje que aparece en la zona de interacción entre las dos placas, y las fuerzas de resistencia al choque (Forsyth and Uyeda, 1975; Zoback, 1992; Strahler, 1992; Heuret and Lallemand, 2005). Se establece que la placa que subduce se mueve con velocidad que sobrepasa la fuerza de fricción generada entre ella y la placa superior, creando una amplia zona de deformación donde se producen los sismos de mayor magnitud. Del mismo modo, como señala Conrad et al. (2004), debido a la fuerza generada por el choque entre las placas, al interior de la placa subducida tiende a ocurrir un debilitamiento que también produce terremotos fuertes que contribuyen a la deformación interna de la misma. A esta zona se atribuyen importantes eventos sísmicos que han causado daño en varias ciudades del suroccidente colombiano, como los ocurridos en noviembre de 1991 y noviembre de 2004.
En el interior del continente se pueden identificar dos aspectos básicos de las implicaciones que tiene el régimen sismotectónico descrito. En primer lugar, la sismicidad de profundidad intermedia producida por la zona de Benioff, cuyos epicentros naturalmente son localizados en la placa continental. Aquí el régimen de esfuerzos es más caótico (Lay et al., 1989) debido a las múltiples fuerzas que tienden a interactuar (Forsyth and Uyeda, 1975; Zoback, 1992), encontrándose entre ellas la misma fuerza de empuje de la dorsal, que es la responsable de la velocidad de subducción de la placa descendente (Capitanio et al., 2007); la fuerza de arrastre del manto que crea una fuerza de presión sobre un lado de la placa en la dirección normal a la fosa; las fuerzas de anclaje natural y la de tracción de la placa (Heuret and Lallemand, 2005).

En segundo lugar, aparecen sismos de carácter superficial, atribuidos a los sistemas de fallas intraplaca presentes en la región. En tal sentido, los eventos que allí se generan como producto del tipo de esfuerzos compresivos o distensivos, aunque históricamente 
no han sido los de mayor liberación de energía en comparación con los eventos producidos en la zona de subducción, su ocurrencia ha causado efectos en la población.

\section{Transferencia de esfuerzos en Eventos de Subducción}

Los terremotos que ocurren en la zonas de subducción son generalmente asociados al descenso de las placas oceánicas en los límites de convergencia (Choy and Kirby, 2004). Lay et al. (1989), argumentan que una de las principales características de la sismicidad en las zonas de subducción radica en el hecho que las réplicas de terremotos fuertes tienen un comportamiento de tipo inverso y tienden a ocurrir debajo de la ruptura de la litosfera y a lo largo de su buzamiento, mientras que las réplicas de tipo normal ocurren antes de la trinchera en su parte superior. Basados en este criterio Lin and Stein (2004), proponen un modelo que establece que un gran terremoto de subducción transfiere su esfuerzo a la corteza circundante, de forma que el fallamiento normal se promueve en la litosfera superior impidiendo el incremento de la ruptura en la zona de Wadati-Benioff. Al contrario, el fallamiento inverso promueve la ruptura en la zona de Wadati-Benioff, pero lo impide en la litosfera superior excepto en una región estrecha del trasarco (backarc).

En el presente trabajo se realizó un modelo de transferencia de esfuerzos en la zona de subducción que abarca el área de estudio, teniendo como base los planos de falla seleccionados de los mecanismos focales de los 49 eventos descritos anteriormente. Para ello se usa el software de sismología estadística ZMAP, del Instituto Federal de Tecnología de Zúrich (ETH) (Wiemer, 2001). Esta herramienta modela un mapa de esfuerzos de Coulomb a partir de la inversión de la orientación del plano de falla (Strike, Slip, Dip-Slip) con base en el método de Michael (1984). En la FIGURA 12, se muestra el mapa de distribución de esfuerzos de Coulomb de la zona de estudio.

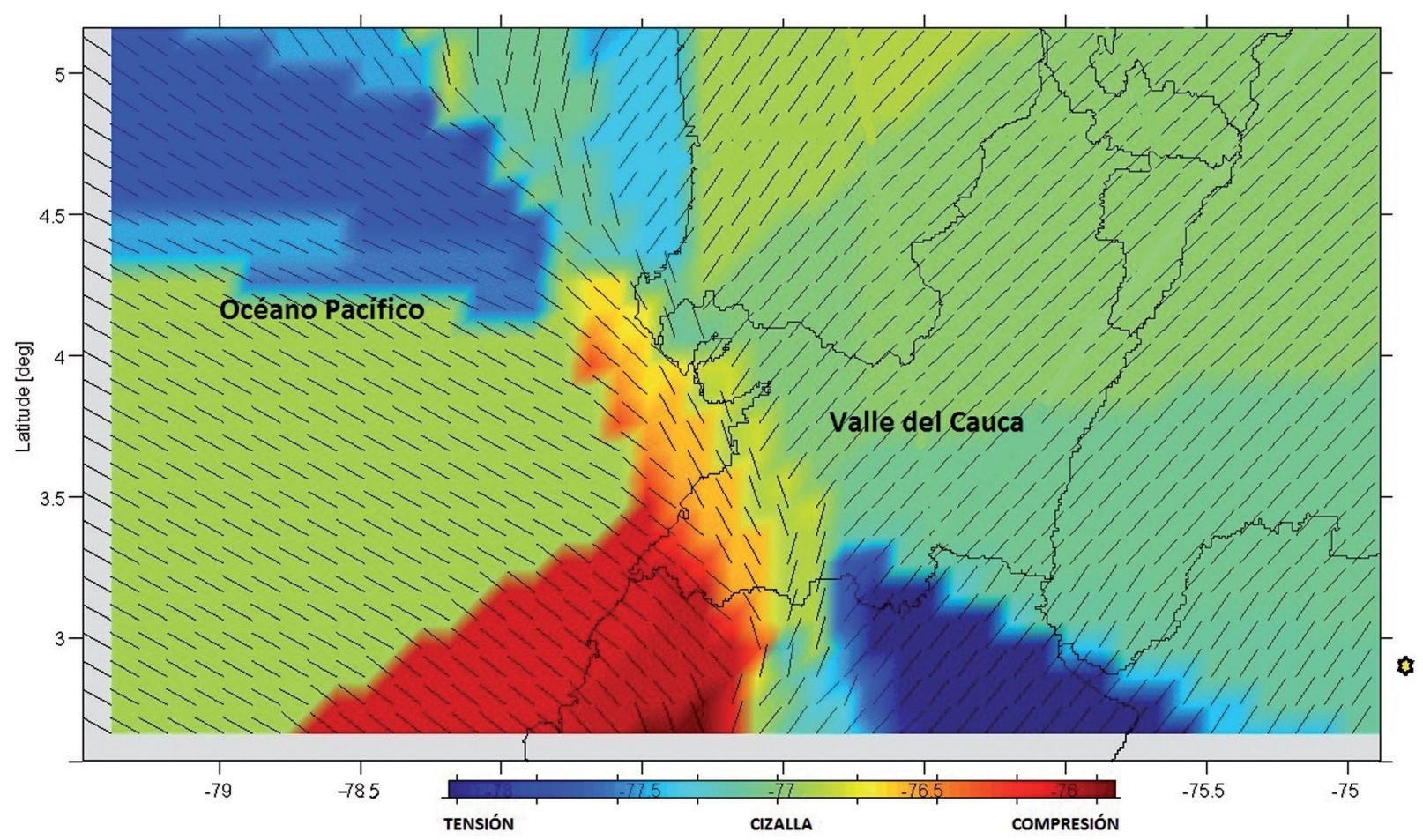

FIGURA 12. Mapa de distribución de esfuerzos sismotectónicos obtenidos a partir de la población de mecanismos focales de los terremotos en la región del Valle del Cauca y zonas aledañas.

Como se puede apreciar en la FIGURA 12, los esfuerzos de compresión se ubican en la zona del litoral pacífico, con orientación SW-NN predominantemente. Este esfuerzo refleja el fuerte contacto entre las placas Nazca y Sudamericana y su fuerte rozamiento al producirse la subducción, no solamente se da en el mar, en la zona de fosa, sino que se prolonga varios kilómetros al interior del continente. Obsérvese como estos esfuerzos no se proyectan en el continente impedidos por esfuerzos de cizallamiento. 
Los esfuerzos de cizalla gobiernan gran parte de la plataforma continental del Valle del Cauca, y se interponen en la proyección de los esfuerzos de tensión que actúan perpendicularmente a los esfuerzos compresivos. En este caso, los esfuerzos compresivos tienen orientación SE-NW, y a diferencia de los esfuerzos compresivos se proyectan en el continente y continúan en la corteza oceánica cerca de la fosa después de haber sido interrumpidos por la superposición de los esfuerzos de cizalla. Como se mencionó anteriormente, dichos esfuerzos impiden la transmisión de los esfuerzos compresivos desde la corteza oceánica a la continental.

El régimen tectónico y la sismicidad de la región del Valle del Cauca y sus zonas aledañas, zona de estudio de este trabajo, es un proceso bastante complejo que se rige principalmente por la interacción de las placas Nazca y Suramericana. El empuje de las fuerzas gravitatorias primordialmente la de empuje desde la dorsal mesooceánica y la de tracción de inmersión que actúa en la placa descendente en la subducción, por un lado, y las fuerzas directamente relacionadas con la fluidez o arrastre del manto junto con las de resistencia entre placas, por el otro, crean un panorama propicio para a la generación de un campo de esfuerzos constante diferencial que se transfiere desde la base del manto hasta la parte superior de la litosfera y desde el océano hasta el continente en todo el sistema de fallas existente.

De esta manera, se considera que esta zona es significativamente importante en la amenaza sísmica de Colombia, ya que en la zona se generan eventos sísmicos fuertes, con magnitudes $M w>6,5$ en intervalos de tiempo de pocos años, que liberan la mayor cantidad de energía elástica acumulada en la región del suroccidente colombiano. Estos eventos causan rupturas sísmicas de gran extensión, como los sismos de 1906 $(M w=8,8), 1942(M w=7,9), 1958(M w=7,7)$ y $1979(M w=8,1)$, que aunque estén fuera de la zona de estudio, han ocasionado graves daños y efectos en la ciudad Santiago de Cali y otras poblaciones importantes de la región.

\section{AGRADECIMIENTOS}

Los autores expresan sus sinceros agradecimientos a los evaluadores anónimos por los comentarios y sugerencias que contribuyeron a mejorar sustancialmente el contenido, presentación y detalles técnicos de nuestro trabajo. Igualmente, al editor del Boletín de Geología Francisco Velandia, por los comentarios constructivos y sugerencias. Esta investigación fue realizada en el marco del proyecto "Caracterización del régimen sísmico y procesos de deformación cortical actual en el Valle del Cauca y zonas aledañas" financiado por la Vicerrectoría de Investigaciones de la Universidad del Valle, registrado con el código CI 297. Agradecemos al Observatorio Sismológico y Geofísico del Suroccidente Colombiano de la Universidad del Valle por el apoyo a este trabajo.

\section{REFERENCIAS}

Acosta, J., Velandia, F., Osorio, J., Lonergan, L. and Mora, H. 2007. Strike-slip deformation within the Colombian Andes. In: Ries, A. C., Butler, R. W. H. and Graham, R. H. (eds). Deformation of the Continental Crust: The Legacy of Mike Coward. Geological Society of London, Special Publications, 272, 303-319.

Adamek, S., Frohlich, C., and Pennington, W.D. 1988. Seismicity of the Caribean-Nazca boundary: constraints on microplate tectonics of the Panama region. Journal of Geophysical Research, 93: 2053-2075.

Aki, K., and Richards, P.G. 1980. Quantitative seismology, theory and methods, Vol. I, W.H. Freeman, San Francisco. 557p.

Arcila, M., y Dimaté, C. 2005. Caracterización de fuentes sísmicas. En: Estudio de microzonificación sísmica de Santiago de Cali. Informe No. 1-6. INGEOMINASDAGMA. M. Bogotá, pp. 38.

Baumbach, M., and Grosser,H. 2009. Seismic sources and source parameters: determination of fault-plane solutions. In: Bormann, P. (Ed.), New manual of seismological observatory practice (NMSOP), Potsdam: Deutsches GeoForschungsZentrum GFZ, pp. 58-70. DOI: http://doi.org/10.2312/GFZ.NMSOP_r1_ch3. Consultado 04-02-2016.

Bormann, P., and Wendt, S. 2013. Determination of fault-plane solutions. En: Bormann, P. (ed). IASPEI, New manual of seismological observatory practice, $2^{\text {nd }}$ Edition (NMSOP; electronic edition). GFZ, Potsdam, Vol. 1, Ch3, pp. 209-225.

Buforn, E. 1994. Métodos para la determinación del mecanismo focal de los terremotos. Física de la Tierra, 6: 113-139.

Buforn, E., y Pro, C. 2006. Mecanismo focal y sismotectónica: aportación de Agustín Udías. Física de la Tierra, 18: 11-23.

Bune, V.I., Balakania, L.M., y Pavlova, G.I. 1975. Sismicidad de Sur América. En: Tectónica, sismicidad y 
geodinámica del sector suroriental del Océano Pacífico (investigaciones geodinámicas). No. 1, pp. 82-99 (en Ruso).

Camacho, E., Hutton, W., and Pacheco, J.F. 2010. A new look evidence for a Wadati-Benioff zone and active convergence at the north Panama deformed belt. Bulletin of the Seismological Society of America, 100(1): 343-348.

Capitanio, F.A., Goes, S., Morra, G., and Giardini, D. 2007. Signatures of downgoing plate-buoyancy driven subduction in motions and seismic coupling at major subduction zones. Earth and Planetary Science Letters, 262: 298-306.

Cardona, C., Salcedo, E., y Mora, H. 2005. Caracterización sismotectónica y geodinámica de la fuente sismogénica de Murindó - Colombia. Boletín de Geología, 27(44): 115-132.

Collot, J.Y., Marcaillou, B., Sage, F., Michaud F., Agudelo, W., Charvis, P., Graindorge, D., Gutscher, M., and Spence, G. 2004. ¿Are rupture zone limits of great subduction earthquakes controlled by upper plate structures? Evidence from multichannel seismic reflection data acquired across the northern EcuadorSouthwest Colombia margin. Journal of Geophysical Research, 109: B11103, doi:10.1029/2004JB003060.

Conrad, C.P., Bilek, S., and Lithgow-Bertelloni, C. 2004. Great earthquakes and slab pull: interaction between seismic coupling and plate slab coupling. Earth and Planetary Science Letters, 218: 109-122.

Corredor, F. 2003. Seismic strain rates and distributed continental deformation in the northern Andes and three-dimensional seismotectonics of northwestern South America. Tectonophysics, 372: 147- 166.

Cortés, M., and Angelier, J. 2005. Current states of stress in the northern Andes as indicated by focal mechanisms of earthquakes. Tectonophysics, 403: 29-58.

Chapple, W., and Forsyth D. 1979. Earthquakes and bending of plates at trenches. Journal of Geophysical Research, 84: 6729-6749.

Choy, G.L., and Kirby, S.H. 2004. Apparent stress, fault maturity and seismic hazard for normal-fault earthquakes at subducction zones. Geophysical Journal International, 159: 991-1012. doi: 10.1111/j.1365246x.2004.02449.x.
Christensen, D., and Ruff, L. 1988. Seismic coupling and outer rise earthquakes, Journal of Geophysical Research, 93: 13421-13444.

Cronin, V.S. 2010. A primer on focal mechanism solutions for geologists. Science Education Resource Center, Carleton College, accessible via http://serc. carleton.edu/files/NAGTWorkshops/structure04/Focal mechanism primer.pdf. Consultado 04-02-2016.

Dziewonski, A.M., Chou, T.A., and Woodhouse, J.H. 1981. Determination of earthquake source parameters from waveform data for studies of global and regional seismicity Journal of Geophysical Research, 86: 2825-2852.

Ekstrom, G., and England, P. 1989. Seismic strain rates in regions of distributed continental deformation. Journal of Geophysical Research, 94: 10231-10257.

Forsyth, D.W., and Uyeda, S. 1975. On the relative importance of the driving forces of plate motion. Geophysical Journal of the Royal Astronomical Society, 43(1): 163-200.

Freymueller, J.T., Kellogg, J.N., and Vega, V. 1993. Plate motions in the North Andean region. Journal of Geophysical Research, 98(B12): 21.853-21.863.

Global CMT Catalog. http://www.globalcmt.org/. Consultado 20-01-2015.

Gutscher, M.A., Malavieille, J., Lallemand, S., and Collot, J.Y. 1999. Tectonic segmentation of the North Andean margin: impact of the Carnegie Ridge collision. Earth and Planetary Science Letters, 168: 255-270.

Guzmán-Speziale, M., and Ni, J.F. 1993. The opening of the Andaman Sea: where is the short-term displacement being taken up? Geophysical Research Letters, 20: 2949-2952.

Guzmán-Speziale, M. 2001. Active seismic deformation in the grabens of northern Central America and its relationship to the relative motion of the North AmericaCaribbean plate boundary. Tectonophysics, 337: 39-51.

Guzmán-Speziale, M., Valdés-González C., Molina E., and Gómez, J.M. 2005. Seismic activity along the Central America volcanic arc: is it related to subduction of the Cocos plate? Tectonophysics, 400: 241- 254.

Herd, D.G., Yound, L., Meyer, H., Arango, J.L., Person, W.J., and Mendoza, C. 1981. The great Tumaco, 
Colombia earthquake of 12 december 1979. Science, 211: 441-445.

Heuret, A., and Lallemand, S. 2005. Plate motions, slab dynamics and back-arc deformation. Physics of the Earth and Planetary Interiors, 149: 31-51.

INGEOMINAS. 2005. Estudio de Microzonificación Sísmica de Cali. Investigaciones Neotectónicas en el Centro Occidente Colombiano. Informe No. 1-3. Bogotá. 171p.

International Seismological Centre (ISC), On-line Bulletin, http://www.isc.ac.uk, Internatl. Seis. Cent., Thatcham, United Kingdom. Consultado 20-01-2015.

Isaacks, B., and Molnar, P. 1969. Mantle earthquakes mechanism and the sinking of the lithosphere. Nature, 223: 1121-1124.

Isaacks, B., Sykes, L.R., and Oliver, J. 1969. Focal mechanisms of deep and shallow earthquakes in the Tonga-Kermadec region and the tectonics of island arcs. Geological Society of America Bulletin, 80: 1443-1470.

Jackson, J., and McKensie, D. 1988. The relationship between plate motions and seismic moment tensor, and rates of active deformation in the Mediterranean and Middle East. Journal of Geophysical Research, 93: 45-73.

Johnson, G.A, Horton, S.P., Withers, M., and Cox, R. 2014. Earthquake Focal Mechanisms in the New Madrid Seismic Zone. Seismological Research Letters, 85(2): 257-267. doi: 10.1785/0220130140.

Jordan, T.H. 1975. The present day motion of the Caribbean plate. Journal of Geophysical Research, 80 (32): 4433-4439.

Kanamori, H., and McNally, K.C. 1982. Variable rupture mode of the subduction zone along the EcuadorColombia Coast. Bulletin of the Seismological Society of America, 72: 1241-1253.

Kelleher J. 1972. Rupture zone of large South American earthquakes and some predictions. Journal of Geophysical Research, 77: 2087-2103.

Kellogg, J.N., Dixon, T., and Neiland, R. 1989. Central and South American GPS Geodesy. EOS Transactions, 70: 649-656.

Kiratzi, A. 1993. A study on the active crustal deformation of the North and East Anatolian Fault Zones. Tectonophysics, 225: 191-203.
Kiratzi, A., and Papazachos, C.B. 1996. MomentoTensor summation to derive active crustal deformation in Japan. Bulletin of the Seismological Society of America, 86: 821-83.

Lay, T., Astiz, L., Kanamori, H., and Christensen, D.H. 1989. Temporal variation of large intraplate earthquakes in coupled subduction zones. Physics of the Earth and Planetary Interiors, 54: 258-312.

Lin, J., and Stein, R.S. 2004. Stress Triggering in thrust and subduction earthquakes and stress interaction between the southern San Andreas and nearby thrust and strike-slip faults. Journal of Geophysical Research, 109: B02303, doi:10.1029/2003JB002607.

Lomnitz, C., and Hashimine, M. 1985. The Popayan, Colombia, earthquake of 31 march 1983. Bulletin of the Seismological Society of America, 75 (5): 1315-1326.

López, A. 2012. Andersonian and Coulomb stresses in central Costa Rica and its fault slip imuttendecy potential: new insigths into their associated seismic hazard. Geological Society of London, Special Publications, 367: 19-38.

López, M.C. 2006. Análisis de deformación tectónica en los piedemontes de las Cordilleras Central y Occidental Valle del Cauca, Colombia. Contribuciones Paleosísmicas. Universidad EAFIT, Medellín, 102p.

Mendiguren, J. 1973. Identification of free oscillation spectral peaks for 1970, July 31, Colombian deep shock using the excitation criterion. Geophysical Journal of the Royal Astronomical Society, 33: 281-321.

Mendoza, C., and Dewey, J. W. 1984. Seismicity associated with the great Colombia-Ecuador earthquakes of 1942, 1958 and 1979: implications for barrier models of earthquakes rupture. Bulletin of the Seismological Society of America, 74 (2): 577-593.

Mezcua, J., Rueda, J., and Buforn, E. 1991. Seismic Deformation in the Azores-Alborean Sea Region. Monografías Instituto Geográfico Nacional, 8: 205-211.

Michael, A. J. 1984. Determination of stress from slip data: Faults and folds. Journal of Geophysical Research, 89: 11517-11526, doi:10.1029/JB089i.

Molnar, P., and Sykes, L.R. 1969.Tectonics of the Caribbean and Middle America regions from focal mechanisms and seismicity. Geological Society of America Bulletin, 80: 1639-1684. 
Monsalve, H. 1998. Geometría de la subducción de la Placa Nazca en el noroeste de Colombia. Universidad Nacional Autónoma de México, México. 107p.

Monsalve, H., y Mora, H. 2005. Esquema geodinámico regional para el noroccidente de Suramérica (Modelo de subducción y desplazamientos relativos). Boletín de Geología, 27 (1): 25-53.

Montes, N., y Sandoval, A. 2001. Base de datos de fallas activas. Recopilación bibliográfica. Proyecto compilación y levantamiento de la información geodinámica. INGEOMINAS. Bogotá.

Murcia, L.A. 1981. Rasgos morfológicos de la tectónica cuaternaria en el suroccidente de Colombia. Geología Norandina, 4: 23-30.

Nivia, A. 2001. Memoria explicativa del mapa geológico del departamento del Valle del Cauca. INGEOMINAS, 148 p.

Nowroozi, A.A. 1972. Focal mechanism of earthquakes in Persia, Turkey, West Pakistan and Afganistan and plate tectonics of the Middle East. Bulletin of the Seismological Society of America, 62: 823-850.

Page, W.D. 1986. Geología sísmica y sismicidad del noroeste de Colombia. ISA, Integral, Woodward-Clyde Consultantes. Medellin, 281p.

Papazachos, C., and Kiratzi, A. 1992. A formulation for reliable estimation of active crustal deformation and its application to central Greece. Geophysical Journal International, 111: 424-432.

París, G., Marín, W., Sauret, B., Vergara, H., y Bles, J. L. 1992. Neotectónica. En: Microzonificación Sismogeotécnica de Popayán. CEE-INGEOMINAS. INGEOMINAS 2: 28-49.

París, G., Machette, M., Dart, R., and Haller, K. 2000. Map and database of Quaternary faults and folds in Colombia and its offshore regions. USGS open-file report 00-0284. Map at 2'500.000 scale and report, 61p.

Pedraza, P. 2006. Geometría de la subducción de la placa Nazca en el suroeste de Colombia, Implicaciones tectónicas y sísmicas. Universidad Nacional de Colombia, Bogotá, 118p.

Pennington, W.D. 1981. Subduction of the eastern Panama Basin and seismotectonics of northwestern South America. Journal of Geophysical Research, 86: 10753-10770.
Pro, C., Buforn, E., and Udías, A. 2007. Rupture length and velocity for earthquakes in the Mid-Atlantic Ridge from directivity effect in body and surface waves. Tectonophysics, 433: 65-79, doi:10.1016/j. tecto.2006.12.011.

Pulido, N. 2003. Seismotectonics of the northern Andes (Colombia) and the development of seismic networks. Bulletin of the International Institute of Seismology and Earthquake Engineering, Special Edition, pp. 69-76.

Rivera, L.A. 1989. Inversion du Tenseur des constraintes et des mécanismes au foyer á partir des donées de polarité pour une population de séismes. Application á I'Etude du foyer de seismicité intermédiaire de Bucaramanga (Colombie). Université Louis-Pasteur de Strasburg, Francia, 266p.

Ruff, L., and Kanamori, H. 1980. Seismicity and the subduction process. Physics of the earth and Planetary Interiors, 23: 240-252, doi:10.1016/00319201(80)90117-X.

Salcedo, E. 1992. Sismicidad y peligro sísmico de Colombia. Universidad Estatal de Moscú "M. V. Lomonosov", Moscú-Rusia, 268p.

Salcedo, E. 1995. Deformación sísmica en las zonas sismoactivas de Chocó y el "Nido" de Bucaramanga (Colombia). Boletín Geológico INGEOMINAS, Bogotá., 35(1): 51-66.

Salcedo, E., Gómez-Capera. A., y Rivera Pérez. C. 1995. Deformación y desplazamiento de bloques corticales en la zona de falla del Borde Llanero colombiano mediante datos sobre mecanismos focales de terremotos. Memorias Seminario de Sismotectónica del Borde Llanero colombiano. Noviembre 9 y 10 de 1995, Bogotá, pp. 66-81.

Salcedo, E., Pérez Rivera, C.I., y Gómez-Capera, A. A. 2001. Implicaciones sismotectónicas de las soluciones del mecanismo focal del algunos terremotos de la región central de Colombia. Revista Geofísica Colombiana, 5: 360-373.

Seno, T., and Yamanaka, Y. 1996. Double seismic zones, compressional deep trench-outer rise events and superplumes. In: Bebout, G. et al., Subduction: top to bottom. Geophysical Monograph Series, 96: 347-355 AGU, Washington, DC.

Sobolieva, O.V. 1990. Deformación de la corteza terrestre durante los terremotos (por ejemplo el valle 
Hissar, Tajikistán). Geodinámica actual y estructura profunda del territorio de la URSS. Nauka. pp. 95-105. (En Ruso).

Strahler, A.N. 1992. Geología Física. Ediciones Omega. Barcelona, 629p.

Sykes, L.R. 1967. Mechanism of earthquakes and nature of faulting on mid-oceanic ridge. Journal of Geophysical Research, 72: 2131-2153.

Tabares, L.M., Mora, H., y Salcedo, E. 1999. Actividad sísmica y tasa de deformación sismotectónica en la zona del Viejo Caldas, Colombia. Academia Colombiana de Ciencias Exactas, Físicas y Naturales, 23(88): 359-373.

Trenkamp, R., Kellogg, J.N., Freymueller, J.T., and Mora, H. 2002. Wide plate margin deformation, southern Central America and northwestern South America, CASA GPS observations. Journal of South American Earth Sciences, 15: 157-171.

Trenkamp, R., Mora, H., Salcedo, E., and Kellogg, J.N. 2004. Possible rapid strain accumulation rates near Cali, Colombia, determined from GPS measurements (19962003). Earth Science Research Journal, 8(1): 25-33.

Udías, A., y Mezcua, J. 1997. Fundamentos de Geofísica. Alianza Editorial S.A. Madrid, 476p.

Udías, A., López-Arroyo, A., and Mezcua, J. 1976. Seismotectonics of the Azores-Alborán region. Tectonophysics, 31: 259-289.

Udías, A., Muñoz, D., y Buforn, E. 1985. Mecanismo de los terremotos y tectónica. Catedra de Geofísica. Facultad de Ciencias Físicas, Universidad Complutense. 232p.

Vargas, C. A., and Mann, P. 2013. Tearing and breaking off of subducted slabs as the results of collision of the Panama Arc-Indenter with northwestern South America. Bulletin of the seismological Society of America, 103(3): 2025-2046. doi: 10.1785/0120120328.

Voronina, E.V., Konov, A.C., and Liucina, A.V. 1990. Cálculo de las componentes de la velocidad media de deformación de la región del arco de islas de Kuriles, Kamchatka y Aleutiana. Física de la Tierra. pp. 7-13. (En Ruso).

Wiemer, S. 2001. A software package to analyze seismicity: ZMAP. Seismological Research Letters, 72: 373-382.
Zoback, M. L. 1992 First- and second-order patterns of stress in the litosphere: the World Stress Map Project. Journal of Geophysical Research, 97: 11703-11728.

\begin{tabular}{c}
\hline \hline Trabajo recibido: agosto 17 de 2014 \\
Trabajo aceptado: mayo 6 de 2016 \\
Manuscrito publicado en internet: mayo 11 de 2016
\end{tabular}

